\title{
A pseudo-monotonicity adapted to doubly nonlinear elliptic-parabolic equations
}

\author{
E. Maitre*, P. Witomski \\ Laboratoire de Modélisation et Calcul, Université de Grenoble I, 38041 Grenoble Cedex, France
}

Received 15 May 2000; accepted 12 February 2001

Keywords: Elliptic-parabolic; Nonlinear; Pseudo-monotonicity; Doubly nonlinear

\section{Introduction}

Let $\mathscr{A}$ and $\mathscr{B}$ be two nonlinear operators over a function space $\mathscr{V}$, with $\mathscr{B}$ possibly multi-valued. We consider the following Cauchy problem: given $T>0, f$ and $v^{0}$, find $u$ such that

$$
\begin{aligned}
& \frac{\mathrm{d}}{\mathrm{d} t} \mathscr{B}(u)+\mathscr{A}(u) \ni f \text { on }[0, T], \\
& \mathscr{B}(u)(0) \ni v^{0} .
\end{aligned}
$$

The case where $\mathscr{B}$ is an unbounded linear operator was considered first by Bardos and Brézis [4]. In the nonlinear case Raviart [20], Grange and Mignot [13], DiBenedetto and Showalter [11] proved existence results assuming that $\mathscr{A}$ and $\mathscr{B}$ are at least monotone operators, and $\mathscr{B}$ is compact. Alt and Luckhaus [1] investigated the case of a non-compact operator $\mathscr{B}$, assuming $\mathscr{A}$ is strongly monotone; their work was extended more recently by Kačur [14], Filo and Kačur [12] or Zadrzyńska and Zajạczkowski [26]. Bermúdez et al. [7] devoted their work to the case where $\mathscr{B}$ is compact and strongly monotone and $\mathscr{A}$ is pseudo-monotone. We are interested in the same case excepted that $\mathscr{B}$ is no longer assumed to be strongly monotone, thus the equation may

\footnotetext{
* Corresponding author. Laboratoire de Mathématiques et Applications, 4 rue des Frères Lumière, Université de Haute Alsace, 68093 Mulhouse, France.

E-mail address: e.maitre@univ-mulhouse.fr (E. Maitre).
} 
degenerate to an elliptic one. Note that in recent results Bénilan and Wittbold [5,6] address this problem using nonlinear semi-group tools. We point out that in the application that motivated our study of such equations (see below), the operator $\mathscr{A}$ depends on time, which makes the semi-group method hard to apply.

Besides its own mathematical interest, this equation arises quite often in diffusion and free boundary problems, as well as in hysteresis operators. In our case, such an equation arises in a mold casting problem. Under physical assumption as the thinness of the mold, one can $[18,17]$ write Navier-Stokes equations as a pressure equation coupled with energy and front propagation equations. It turns out that this pressure equation can be rewritten under (EP) form, with explicit dependence of the elliptic part on $u$ (thanks to a suitable change of unknown):

$$
\frac{\partial \beta(u)}{\partial t}-\operatorname{div}(\beta(u) S(x, t, u) \nabla u)=f(x, t) .
$$

The structure of our article is the following: in Sections 2 and 3 we define and study a new class of operators ( $\mathscr{B}$-pseudo-monotone) for which we state in Section 3 an existence result, under given assumptions on the data. We operate a time discretization of (EP) which leads to a variational inequality solved thanks to a result of Lions. We then derive a priori estimates and prove a nonlinear compactness lemma to pass to the limit in nonlinear terms. This compactness lemma is a nonlinear counterpart of known results of Simon (see Remark 3). As an application, we give a set of growth and monotonicity conditions adapted from the variational operators of Lions, which form a sub-class of $\mathscr{B}$-pseudo-monotone operators, in which falls our physical operator. The last Section consists of two technical lemmas which have been postponed during the existence proof for sake of readability.

\section{Definitions and notations}

\subsection{Functional spaces}

Let $V$ and $W$ be two separable and reflexive Banach spaces, such that $V$ is dense and compactly embedded in $W$. We denote this injection by $i$, and its dual operator by $i^{*}$. Let us introduce for $T>0$

$$
\left.\mathscr{V}=L^{p}(0, T ; V) \quad \text { and } \quad \mathscr{W}=L^{p}(0, T ; W) \quad \text { where } p \in\right] 1,+\infty[.
$$

We denote by $\langle$,$\rangle and (, ) the duality brackets of V^{\prime} \times V$ and $\mathscr{V}^{\prime} \times \mathscr{V}$ respectively. $q$ stands for the conjugate exponent of $p$.

\subsection{Pseudo-monotonicity}

In order to define a pseudo-monotonicity adapted to nonlinear elliptic-parabolic equations, let us consider them as intermediate equations between elliptic and parabolic equations, and try to understand how the notion of pseudo-monotonicity has been devised for these two cases. 
Let $\mathscr{A}$ be a bounded and coercive operator from $\mathscr{V}$ to $\mathscr{V}^{\prime}$, and consider the following elliptic problem:

$$
\begin{aligned}
& \text { Given } f \in \mathscr{V}^{\prime} \text {, find } u \in \mathscr{V} \text { such that } \\
& \mathscr{A}(u)=f .
\end{aligned}
$$

A quite classical method to study this problem is to reduce it to a finite dimensional problem using a Galerkin method, the main difficulty being to pass to the limit in non linear terms:

$$
\mathscr{A}\left(u_{n}\right) \rightarrow \mathscr{A}(u)
$$

since we only have in general a weak convergence of $u_{n}$. The standard method uses a priori estimates to get

$$
\limsup _{n \rightarrow \infty}\left(\mathscr{A}\left(u_{n}\right), u_{n}-u\right) \leqslant 0 .
$$

If the structure of $\mathscr{A}$ is such that this condition implies

$$
\liminf _{n \rightarrow \infty}\left(\mathscr{A}\left(u_{n}\right), u_{n}-v\right) \geqslant(\mathscr{A}(u), u-v), \quad \forall v \in \mathscr{V},
$$

then one easily shows that (1) is verified [16, p. 180]. As a matter of fact, Lions proves existence of a solution to (E) when $\mathscr{A}$ is pseudo-monotone on $\mathscr{V}$, that is when for each sequence $u_{n}$ weakly convergent to $u$ in $\mathscr{V}$, (2) implies (3).

On the other side, for the following parabolic problem:

Let $f \in \mathscr{V}^{\prime}$ and $u_{0} \in V$, find $u \in \mathscr{V}$ such that

$$
\frac{\mathrm{d} u}{\mathrm{~d} t}+\mathscr{A}(u)=f, \quad(u)(0)=u_{0},
$$

one usually gets the convergence of $u_{n}^{\prime}$. Following Lions, (P) has a solution if $\mathscr{A}$ is supposed to be pseudo-monotone on $D(\mathrm{~d} / \mathrm{d} t)$, i.e. for each sequence $u_{n}$ weakly convergent to $u$ in $\mathscr{V}$ such that $u_{n}^{\prime}$ weakly converges to $u^{\prime}$ in $\mathscr{V}^{\prime}$, from (2) follows that (3) holds.

Consider a bounded nonlinear operator $\mathscr{B}$ from $\mathscr{V}$ to $\mathscr{W}^{\prime}$. We try to define a pseudo-monotonicity for the following problem:

$$
\begin{aligned}
& \text { Given } f \in \mathscr{V}^{\prime} \text { and } v_{0} \in W^{\prime}, \text { find } u \in \mathscr{V} \text { such that } \\
& \frac{\mathrm{d}}{\mathrm{d} t} \mathscr{B}(u)+\mathscr{A}(u)=f, \quad \mathscr{B}(u)(0)=v_{0} .
\end{aligned}
$$

Analogously one could be tempted to replace in the pseudo-monotonicity definition the weak convergence of $u_{n}^{\prime}$ by those of $\left(\mathscr{B}\left(u_{n}\right)\right)^{\prime}$ in $\mathscr{V}^{\prime}$. However, when the passing to the limit is performed in $\mathscr{A}$, convergence of $u_{n}^{\prime}$ is indirectly used to prove the strong convergence of a subsequence of $u_{n}$ in $\mathscr{W}$ (owing to an Aubin-type lemma [16, p. 57]). The only assumption of weak convergence of $\left(\mathscr{B}\left(u_{n}\right)\right)^{\prime}$ in $\mathscr{V}^{\prime}$ would not lead to a strong convergence of $\mathscr{B}\left(u_{n}\right)$ in a sufficiently regular functions space (for example $\mathscr{W}^{\prime}$ ).

Thus we were naturally lead to introduce the following definition. 
Definition 1. An operator $\mathscr{A}$ is $\mathscr{B}$-pseudo-monotone if it is bounded and for any sequence $u_{n}$ weakly convergent to $u$ in $\mathscr{V}$, such that $\mathscr{B}\left(u_{n}\right)$ strongly converges to $\mathscr{B}(u)$ in $\mathscr{W}^{\prime}$, the condition

$$
\limsup _{n \rightarrow \infty}\left(\mathscr{A}\left(u_{n}\right), u_{n}-u\right) \leqslant 0
$$

implies

$$
\liminf _{n \rightarrow \infty}\left(\mathscr{A}\left(u_{n}\right), u_{n}-v\right) \geqslant(\mathscr{A}(u), u-v), \quad \forall v \in \mathscr{V} .
$$

\section{Properties of $\mathscr{B}$-pseudo-monotone operators}

We verify in this section that we actually defined an intermediate notion between pseudo-monotonicity on $\mathscr{V}$ and those introduced by Lions for parabolic problems. Moreover, we show an additivity property in this class.

Proposition 1. (i) A pseudo-monotone operator on $\mathscr{V}$ (a fortiori hemicontinuous monotone) is $\mathscr{B}$-pseudo-monotone on $\mathscr{V}$, for any operator $\mathscr{B}$.

(ii) If $\mathscr{B}$ is compact from $\mathscr{V}$ to $\mathscr{W}^{\prime}$ then $\mathscr{B}$-pseudo-monotonicity coincides with pseudo-monotonicity on $\mathscr{V}$.

(iii) An operator $\mathscr{B}$-pseudo-monotone, with $\mathscr{B}$ continuous from $\mathscr{W}$ to $\mathscr{W}^{\prime}$ is pseudo-monotone in the parabolic sense, i.e. on the space $W(0, T)=\left\{u \in \mathscr{V}: u^{\prime} \in \mathscr{V}^{\prime}\right\}$.

(iv) For any $\mathscr{B}$ operator from $\mathscr{V}$ to $\mathscr{W}^{\prime}$, the sum of two $\mathscr{B}$-pseudo-monotone operators is $\mathscr{B}$-pseudo-monotone.

Proof. The first two points are easily deduced from definition. To show the third one, let us take an operator $\mathscr{A}$ which is $\mathscr{B}$-pseudo-monotone on $\mathscr{V}$. Let us denote by $\left(u_{n}\right) \subset \mathscr{V}$ a sequence converging weakly to $u$ in $W(0, T)$, verifying

$$
\limsup _{n \rightarrow \infty}\left(\mathscr{A}\left(u_{n}\right), u_{n}-u\right) \leqslant 0 .
$$

The compactness result of [22] (Corollary 4, p. 85) implies strong convergence of a subsequence $\left(u_{m}\right)$ in $\mathscr{W}$. The continuity assumption on $\mathscr{B}$ implies

$$
\mathscr{B}\left(u_{m}\right) \rightarrow \mathscr{B}(u) \text { strongly in } \mathscr{W}^{\prime} .
$$

For this subsequence, we still have

$$
\limsup _{m \rightarrow \infty}\left(\mathscr{A}\left(u_{m}\right), u_{m}-u\right) \leqslant 0,
$$

thus from the definition of $\mathscr{B}$-pseudo-monotonicity

$$
\liminf _{m \rightarrow \infty}\left(\mathscr{A}\left(u_{m}\right), u_{m}-v\right) \geqslant(\mathscr{A}(u), u-v), \quad \forall v \in \mathscr{V} .
$$

But this properties holds for the whole sequence $\left(u_{n}\right)$. Otherwise, one could extract a subsequence from $\left(u_{n}\right)$ which would not verify this property, and the same procedure on this subsequence would lead to a contradiction. Finally $\mathscr{A}$ is pseudo-monotone on $W(0, T)$. 
At last, let us consider two $\mathscr{B}$-pseudo-monotone operators $\mathscr{A}_{1}, \mathscr{A}_{2}$. Let $\mathscr{A}=\mathscr{A}_{1}+$ $\mathscr{A}_{2}$, and take a sequence $u_{n}$, weakly convergent to $u$ in $\mathscr{V}$, such that $\mathscr{B}\left(u_{n}\right)$ strongly converges to $\mathscr{B}(u)$ in $\mathscr{W}^{\prime}$ and

$$
\limsup _{n \rightarrow \infty}\left(\mathscr{A}\left(u_{n}\right), u_{n}-u\right) \leqslant 0 .
$$

We claim that it implies

$$
\limsup _{n \rightarrow \infty}\left(\mathscr{A}_{1}\left(u_{n}\right), u_{n}-u\right) \leqslant 0 \text { and } \limsup _{n \rightarrow \infty}\left(\mathscr{A}_{2}\left(u_{n}\right), u_{n}-u\right) \leqslant 0 .
$$

Otherwise, we can pick a subsequence still denoted by $\left(u_{n}\right)$, such that

$$
\lim _{n \rightarrow \infty}\left(\mathscr{A}_{1}\left(u_{n}\right), u_{n}-u\right)=a>0
$$

and thus

$$
\limsup _{n \rightarrow \infty}\left(\mathscr{A}_{2}\left(u_{n}\right), u_{n}-u\right) \leqslant-a .
$$

For this subsequence $\mathscr{B}\left(u_{n}\right)$ still converges to $\mathscr{B}(u)$, and from the $\mathscr{B}$-pseudo-monotonicity of $\mathscr{A}_{2}$,

$$
\liminf _{n \rightarrow \infty}\left(\mathscr{A}_{2}\left(u_{n}\right), u_{n}-v\right) \geqslant\left(\mathscr{A}_{2}(u), u-v\right), \quad \forall v \in \mathscr{V} .
$$

In particular, for $v=u$ we contradict $a>0$. Thus (4) holds, and as $\mathscr{A}_{1}$ and $\mathscr{A}_{2}$ are $\mathscr{B}$-pseudo-monotone,

$$
\begin{aligned}
& \liminf _{n \rightarrow \infty}\left(\mathscr{A}_{1}\left(u_{n}\right), u_{n}-v\right) \geqslant\left(\mathscr{A}_{1}(u), u-v\right), \quad \forall v \in \mathscr{V}, \\
& \liminf _{n \rightarrow \infty}\left(\mathscr{A}_{2}\left(u_{n}\right), u_{n}-v\right) \geqslant\left(\mathscr{A}_{2}(u), u-v\right), \quad \forall v \in \mathscr{V} .
\end{aligned}
$$

The proof is ended owing to the sup-additivity of the inferior limit,

$$
\begin{aligned}
\liminf _{n \rightarrow \infty}\left(\mathscr{A}\left(u_{n}\right), u_{n}-u\right) & \geqslant \liminf _{n \rightarrow \infty}\left(\mathscr{A}_{1}\left(u_{n}\right), u_{n}-u\right)+\liminf _{n \rightarrow \infty}\left(\mathscr{A}_{2}\left(u_{n}\right), u_{n}-u\right) \\
& \geqslant(\mathscr{A}(u), u-v), \quad \forall v \in \mathscr{V} . \quad \square
\end{aligned}
$$

Remark 1. We will prove in Section 4.7 that classical variational operators as introduced in [16, p. $182-187,321-325]$, are $\mathscr{B}$-pseudo-monotone for an operator $\mathscr{B}$ strictly monotone.

\section{Assumptions and results}

We now turn to the study of the following nonlinear evolution equation:

$$
\begin{aligned}
& \frac{\mathrm{d}}{\mathrm{d} t} \mathscr{B}(u)+\mathscr{A}(u) \ni f, \\
& \mathscr{B}(u)(0)=v^{0} .
\end{aligned}
$$


Let us make more precise which are our operators and assumptions made on them. We consider a convex lower semi-continuous and proper functional $\Phi$ on $W$, such that

$\Phi$ is finite and continuous on $i(V)$, with $\Phi(0)=0$,

$$
\exists C>0, \forall u \in V, \quad\|\partial \Phi \circ i(u)\|_{W^{\prime}} \leqslant C\left(1+\|u\|_{V}^{p-1}\right) .
$$

We then define $B=\partial(\Phi \circ i)=i^{*} \circ \partial \Phi \circ i$, which is maximal monotone from $V$ to $V^{\prime}$. Operator $\mathscr{B}$ from $\mathscr{V}$ to $\mathscr{V}^{\prime}$ is constructed as follows:

$$
v \in \mathscr{B}(u) \Leftrightarrow v(t) \in B(u(t)) \text { a.e. on }[0, T] \text {. }
$$

By this construction and owing to (6), this operator is maximal monotone and bounded from $\mathscr{V}$ to $\mathscr{W}^{\prime}$.

We now consider a family $\{A(t,),. t \in[0, T]\}$ of operators from $V$ to $V^{\prime}$ verifying the following assumption

$$
\exists C>0, \forall v \in V, \quad\|A(t, v)\|_{V^{\prime}} \leqslant C\left(1+\|v\|_{V}^{p-1}\right) \quad \text { a.e. on }[0, T] .
$$

Analogously we define $\mathscr{A}: \mathscr{V} \rightarrow \mathscr{V}^{\prime}$ by

$$
\forall u \in \mathscr{V}, \quad \mathscr{A}(u)(t)=A(t, u(t)) \quad \text { a.e. on }[0, T]
$$

and assume

$$
\liminf _{\|u\|_{\mathscr{r}} \rightarrow \infty} \frac{(\mathscr{A}(u), u)}{\|u\|_{\mathscr{r}}^{p}}>0 .
$$

We can now state our main result:

Theorem 1. Let $f \in \mathscr{V}^{\prime}$, and $v^{0} \in D\left((\Phi \circ i)^{*}\right)$. Under assumptions (5)-(8), provided $\mathscr{A}$ is pseudo-monotone on $\mathscr{V}$, or $\mathscr{B}$-pseudo-monotone with $\mathscr{B}$ continuous from $\mathscr{W}$ to $\mathscr{W}^{\prime}$ for the strong topology, there exists $u \in \mathscr{V}$ and $v \in \mathscr{W}^{\prime} \cap L^{\infty}\left(0, T ; V^{\prime}\right)$ such that $\mathrm{d} v / \mathrm{d} t \in \mathscr{V}^{\prime}$ and:

$$
\text { (E) }\left\{\begin{array}{l}
\frac{\mathrm{d} v}{\mathrm{~d} t}+\mathscr{A}(u)=f, \\
v \in \mathscr{B}(u), \\
v(0)=v^{0} .
\end{array}\right.
$$

This solution verifies $v(t) \in D\left((\Phi \circ i)^{*}\right)$ for all $t \in[0, T]$. Moreover, if $\Phi$ is continuous on $W$ then $v \in L^{\infty}\left(0, T ; W^{\prime}\right)$.

\section{Remark 2}

- The initial condition on $v$ has a meaning since $v \in \mathscr{W}$ and $\frac{\mathrm{d} v}{\mathrm{~d} t} \in \mathscr{V}^{\prime}$ imply $v \in$ $C\left(0, T ; V^{\prime}\right)$ [27, vol. IIA, p. 446],

- The previous theorem gives as particular cases the elliptic and parabolic existence results of Lions: for $\Phi=0$ we have $D\left((\Phi \circ i)^{*}\right)=\{0\}$ and $\mathscr{B}$-pseudo-monotonicity coincide with elliptic pseudo-monotonicity. For $\Phi(u)=\frac{1}{2}\|u\|_{W}^{2}$ we have $D\left((\Phi \circ i)^{*}\right)$ $=W$.

- If $\mathscr{B}$ is continuous from $\mathscr{W}$ in $\mathscr{W}^{\prime}$ then $B$ is also continuous from $W$ to $W^{\prime}$. 
- Observe that $v(t)$ remains for all time in the same set as the initial condition. That could be used for the case where $B$ depends on times explicitly.

- Our assumption on $B$ is weaker than those of [7]. As a counterpart, the coerciveness assumption on $\mathscr{A}$ is stronger, since for simplicity $B$ does not play a role at this level. However, we could think to weaken assumption (8) using the contribution of the functional $\Phi$ or operator $B$.

To prove Theorem 1, we operate a time discretization. Then we write the obtained stationary problem as a variational inequality and apply an existence result of [16]. We obtain a priori estimates which permit us to pass to the limit in the case where $\mathscr{A}$ is pseudo-monotone in the usual sense. We show a compactness lemma which allows the passing to the limit in the case of a $\mathscr{B}$-pseudo-monotone operator.

\subsection{Time discretization of $(\mathrm{E})$}

Let $N$ be an integer intended for going to infinity, and a subdivision $\left(t_{i}\right)_{0 \leqslant i \leqslant N}$ of $[0, T]$ whose step is $\varepsilon=T / N$. As in [7], we consider the discretized problem:

Find $\left(u_{\varepsilon}^{0}, \ldots, u_{\varepsilon}^{N}\right) \in V^{N+1}$ such that

$$
\left(P_{\varepsilon}\right) \begin{cases}\frac{v_{\varepsilon}^{n+1}-v_{\varepsilon}^{n}}{\varepsilon}+A_{\varepsilon}^{n}\left(u_{\varepsilon}^{n+1}\right)=f_{\varepsilon}^{n} & \text { for } n=0, \ldots, N-1, \\ v_{\varepsilon}^{n+1} \in B\left(u_{\varepsilon}^{n+1}\right) & \text { for } n=0, \ldots, N-1, \\ v_{\varepsilon}^{0}=v^{0}, & \end{cases}
$$

where we set

$$
\| \begin{aligned}
f_{\varepsilon}^{n}=\frac{1}{\varepsilon} \int_{t_{n}}^{t_{n+1}} f(t) \mathrm{d} t, \\
A_{\varepsilon}^{n}: V \rightarrow V^{\prime} \\
\quad u \rightarrow A_{\varepsilon}^{n} u=\frac{1}{\varepsilon} \int_{t_{n}}^{t_{n+1}} A(t, u) \mathrm{d} t .
\end{aligned}
$$

A first lemma tells us about the behavior of $f_{\varepsilon}^{n}$ :

Lemma 1. Let $X$ be a separable and reflexive Banach space, and $f \in L^{p}(0, T ; X)$. For each $\varepsilon>0, f_{\varepsilon}$ stands for the step function on $[0, T]$ such that

$$
f_{\varepsilon}(t)=\frac{1}{\varepsilon} \int_{t_{n}}^{t_{n+1}} f(s) \mathrm{d} s \quad \text { for } t \in\left[t_{n}, t_{n+1}[.\right.
$$

Then

$$
f_{\varepsilon} \rightarrow f \quad \text { strongly in } L^{p}(0, T ; X) .
$$

Proof. We first show that

$$
\forall \varepsilon>0, \forall f \in L^{p}(0, T ; X), \quad\left\|f_{\varepsilon}\right\| \leqslant\|f\|,
$$

and then that the lemma holds for continuous functions on $[0, T]$. The lemma follows by a density argument. 
Let us study the properties of operator $A_{\varepsilon}^{n}$ :

Lemma 2. Under the assumptions of Theorem 1 on $\mathscr{A}, A_{\varepsilon}^{n}$ verifies

(i) $\exists C>0, \forall v \in V,\left\|A_{\varepsilon}^{n} v\right\|_{V^{\prime}} \leqslant C\left(1+\|v\|_{V}^{p-1}\right)$,

(ii) $A_{\varepsilon}^{n}$ is pseudo-monotone from $V$ to $V^{\prime}$,

(iii) $\liminf _{\|v\|_{V} \rightarrow \infty} \frac{\left\langle A_{\varepsilon}^{n} v, v\right\rangle}{\|v\|_{V}^{p}}>0$.

(iv) There exist two constants $\alpha, \gamma>0$ independent of $s$ and $\varepsilon$ and such that

$$
\varepsilon \sum_{n=0}^{s}\left\langle A_{\varepsilon}^{n} u_{\varepsilon}^{n+1}, u_{\varepsilon}^{n+1}\right\rangle \geqslant \alpha \varepsilon \sum_{n=0}^{s}\left\|u_{\varepsilon}^{n+1}\right\|_{V}^{p}-\gamma, \quad \forall s \in\{0, \ldots, N-1\} .
$$

Proof. To show (i) it suffices to integrate (7) on $\left[t_{n}, t_{n+1}\right]$.

In order to get (ii), take a sequence $u_{m}$ converging weakly towards $u$ and assume that

$$
\limsup _{m \rightarrow \infty}\left\langle A_{\varepsilon}^{n} u_{m}, u_{m}-u\right\rangle \leqslant 0 .
$$

The function $\tilde{u}_{m}$ belonging to $\mathscr{V}$ and defined by

$$
\tilde{u}_{m}(t)= \begin{cases}u_{m} & \text { if } t \in\left[t_{n}, t_{n+1}\right], \\ u & \text { elsewhere on }[0, T],\end{cases}
$$

weakly converges to the function $\tilde{u}$ of $\mathscr{V}$ which is equal to $u$ on $[0, T]$.

For this sequence, the inequality

$$
\limsup _{m \rightarrow \infty}\left\langle A_{\varepsilon}^{n} u_{m}, u_{m}-u\right\rangle \leqslant 0
$$

means

$$
\limsup _{m \rightarrow \infty}\left(\mathscr{A} \tilde{u}_{m}, \tilde{u}_{m}-\tilde{u}\right) \leqslant 0 .
$$

Case 1: If $\mathscr{A}$ is pseudo-monotone on $\mathscr{V}$, then we get from the definition

$$
\liminf _{m \rightarrow \infty}\left(\mathscr{A} \tilde{u}_{m}, \tilde{u}_{m}-\tilde{v}\right) \geqslant(\mathscr{A} \tilde{u}, \tilde{u}-\tilde{v}) \quad \forall \tilde{v} \in \mathscr{V} .
$$

Case 2: If $\mathscr{A}$ is only $\mathscr{B}$-pseudo-monotone, the compact embedding of $V$ in $W$ allows us to extract from the sequence $\left(u_{m}\right)$ a subsequence strongly convergent to $u$ in $W$; this implies the strong convergence of a subsequence $\tilde{u}_{\sigma(m)}$ towards $\tilde{u}$ in $\mathscr{W}$. From the continuity assumption on $\mathscr{B}$ we deduce the strong convergence of $\mathscr{B}\left(\tilde{u}_{\sigma(m)}\right)$. Thus we still have (9) for the subsequence $\sigma(m)$. By a standard argument the whole sequence verifies (9).

In particular, for any $v \in V$ and $\tilde{v} \in \mathscr{V}$ equal to $v$ on $\left[t_{n}, t_{n+1}\right]$ and $u$ elsewhere in $[0, T]$, we get

$$
\liminf _{m \rightarrow \infty}\left\langle A_{\varepsilon}^{n} u_{m}, u_{m}-v\right\rangle \geqslant\left\langle A_{\varepsilon}^{n} u, u-v\right\rangle \quad \forall v \in V .
$$

Thus (ii) is proved.

Property (iii) is obtained in a similar way, considering in (8) a function $\tilde{u}$ belonging to $\mathscr{V}$, with value $u \in V$ on $\left[t_{n}, t_{n+1}\right]$ and 0 elsewhere on $[0, T]$. 
At last we prove (iv) using (7) and (8). Indeed, if $2 \alpha>0$ denotes the inferior limit appearing in (8), we have

$$
\exists d>0, \forall u \in \mathscr{V}, \quad\|u\|_{\mathscr{V}} \geqslant d \Rightarrow \frac{(\mathscr{A}(u), u)}{\|u\|_{\mathscr{V}}^{p}} \geqslant \alpha,
$$

and (7) shows us that there exists $\gamma_{1}>0$ such that

$$
\forall u \in \mathscr{V}, \quad\|u\|_{\mathscr{V}} \leqslant d \Rightarrow|(\mathscr{A}(u), u)| \leqslant \gamma_{1} .
$$

Thus there exists $\gamma_{2}>0$ such that

$$
\forall u \in \mathscr{V}, \quad(\mathscr{A}(u), u) \geqslant \alpha\|u\|_{\mathscr{V}}^{p}-\gamma_{2} .
$$

In particular if $u \in \mathscr{V}$ equals $u_{\varepsilon}^{n+1}$ on $\left.] t_{n}, t_{n+1}\right]$ for $n=0 \ldots s$, with $s \in\{0, \ldots, N-1\}$, we get (iv).

Lemma 3. The discretized problem $\left(\mathrm{P}_{\varepsilon}\right)$ has a solution.

Proof. This discretized problem may also be written as

Find $u_{\varepsilon}^{n+1}$ such that

$$
f_{\varepsilon}^{n}-A_{\varepsilon}^{n} u_{\varepsilon}^{n+1}+\frac{1}{\varepsilon} v_{\varepsilon}^{n} \in \frac{1}{\varepsilon} B\left(u_{\varepsilon}^{n+1}\right) .
$$

From the definition of sub-differential, this last relation is equivalent to

$$
\left\langle A_{\varepsilon}^{n} u_{\varepsilon}^{n+1}, v-u_{\varepsilon}^{n+1}\right\rangle+\frac{1}{\varepsilon} \Phi \circ i(v)-\frac{1}{\varepsilon} \Phi \circ i\left(u_{\varepsilon}^{n+1}\right) \geqslant\left\langle f_{\varepsilon}^{n}+\frac{1}{\varepsilon} v_{\varepsilon}^{n}, v-u_{\varepsilon}^{n+1}\right\rangle \quad \forall v \in V .
$$

We now use the following result on elliptic variational inequalities: (cf [16, p. 251] see also [8, p. 138]):

Theorem 2. Let $A$ be a pseudo-monotone operator from $V$ to $V^{\prime}, \Psi$ a proper lower semi-continuous convex functional. Assume that

there exists $v_{0}$ such that $\Psi\left(v_{0}\right)<\infty$ and

$$
\frac{\left\langle A(u), u-v_{0}\right\rangle+\Psi(u)}{\|u\|_{V}} \rightarrow \infty \quad \text { whenever }\|u\|_{V} \rightarrow \infty
$$

Then, for a given $f$ in $V^{\prime}$, there exists $u \in V$ solution of

$$
\langle A(u)-f, v-u\rangle+\Psi(v)-\Psi(u) \geqslant 0 \quad \forall v \in V .
$$

We can use this result for $v_{0}=0$, provided we show that

$$
\lim _{\|u\|_{V} \rightarrow \infty} \frac{\left\langle A_{\varepsilon}^{n} u, u\right\rangle+(1 / \varepsilon) \Phi \circ i(u)}{\|u\|_{V}}=+\infty .
$$

In fact we can show more. Indeed under our assumptions, $\Phi \circ i$ is bounded from below since

$$
\forall u \in V, \forall v_{0} \in B(0), \quad \Phi \circ i(u)-\Phi \circ i(0) \geqslant\left\langle v_{0}, u\right\rangle .
$$


Using Lemma 2 we thus get

$$
\liminf _{\|u\|_{V} \rightarrow \infty} \frac{\left\langle A_{\varepsilon}^{n} u, u\right\rangle+(1 / \varepsilon) \Phi \circ i(u)}{\|u\|_{V}^{p}}>0,
$$

from which (10) follows since $p>1$.

\subsection{A priori estimates}

Consider for $n \in\{0, \ldots, N-1\}$ the equations of $\left(\mathrm{P}_{\varepsilon}\right)$

$$
\frac{v_{\varepsilon}^{n+1}-v_{\varepsilon}^{n}}{\varepsilon}+A_{\varepsilon}^{n}\left(u_{\varepsilon}^{n+1}\right)=f_{\varepsilon}^{n}
$$

and take $u_{\varepsilon}^{n+1}$ as a test function. We get

$$
\frac{1}{\varepsilon}\left\langle v_{\varepsilon}^{n+1}-v_{\varepsilon}^{n}, u_{\varepsilon}^{n+1}\right\rangle+\left\langle A_{\varepsilon}^{n} u_{\varepsilon}^{n+1}, u_{\varepsilon}^{n+1}\right\rangle=\left\langle f_{\varepsilon}^{n}, u_{\varepsilon}^{n+1}\right\rangle .
$$

But $u_{\varepsilon}^{n+1} \in \partial(\Phi \circ i)^{*}\left(v_{\varepsilon}^{n+1}\right)$ and the convexity of $(\Phi \circ i)^{*}$ gives

$$
\left\langle v_{\varepsilon}^{n+1}-v_{\varepsilon}^{n}, u_{\varepsilon}^{n+1}\right\rangle \geqslant(\Phi \circ i)^{*}\left(v_{\varepsilon}^{n+1}\right)-(\Phi \circ i)^{*}\left(v_{\varepsilon}^{n}\right) .
$$

Summing from $n=0$ to $s \in\{0, \ldots, N-1\}$, we get

$$
(\Phi \circ i)^{*}\left(v_{\varepsilon}^{s+1}\right)+\varepsilon \sum_{n=0}^{s}\left\langle A_{\varepsilon}^{n} u_{\varepsilon}^{n+1}, u_{\varepsilon}^{n+1}\right\rangle \leqslant \varepsilon \sum_{n=0}^{s}\left\langle f_{\varepsilon}^{n}, u_{\varepsilon}^{n+1}\right\rangle+(\Phi \circ i)^{*}\left(v^{0}\right) .
$$

We point out that the assumption $\Phi(0)=0$ implies the positiveness of $(\Phi \circ i)^{*}$ on $V^{\prime}$; using Lemma 2, (iv) we get

$$
\alpha \varepsilon \sum_{n=0}^{s}\left\|u_{\varepsilon}^{n+1}\right\|_{V}^{p} \leqslant \varepsilon \sum_{n=0}^{s}\left\langle f_{\varepsilon}^{n}, u_{\varepsilon}^{n+1}\right\rangle+(\Phi \circ i)^{*}\left(v^{0}\right)+\gamma, \quad \forall s \in\{0, \ldots, N-1\} .
$$

Let us first work on the right hand side, applying Hölder inequality:

$$
\begin{aligned}
\varepsilon\left|\sum_{n=0}^{s}\left\langle f_{\varepsilon}^{n}, u_{\varepsilon}^{n+1}\right\rangle\right| & \leqslant \varepsilon \sum_{n=0}^{s}\left\|f_{\varepsilon}^{n}\right\|_{V^{\prime}}\left\|u_{\varepsilon}^{n+1}\right\|_{V} \\
& \leqslant \varepsilon\left(\sum_{n=0}^{s}\left\|f_{\varepsilon}^{n}\right\|_{V^{\prime}}^{q}\right)^{1 / q}\left(\sum_{n=0}^{s}\left\|u_{\varepsilon}^{n+1}\right\|_{V}^{p}\right)^{1 / p} \\
& \leqslant\left(\varepsilon \sum_{n=0}^{N-1}\left\|f_{\varepsilon}^{n}\right\|_{V^{\prime}}^{q}\right)^{1 / q}\left(\varepsilon \sum_{n=0}^{s}\left\|u_{\varepsilon}^{n+1}\right\|_{V}^{p}\right)^{1 / p} \\
& \leqslant\left\|f_{\varepsilon}\right\|_{\mathscr{V}^{\prime}}\left(\varepsilon \sum_{n=0}^{s}\left\|u_{\varepsilon}^{n+1}\right\|_{V}^{p}\right)^{1 / p} \\
& \leqslant\|f\|_{\mathscr{V}^{\prime}}\left(\varepsilon \sum_{n=0}^{s}\left\|u_{\varepsilon}^{n+1}\right\|_{V}^{p}\right)^{1 / p}
\end{aligned}
$$


the last inequality being obtained from the demonstration of Lemma 1. Finally, we have

$$
\begin{aligned}
& \alpha \varepsilon \sum_{n=0}^{s}\left\|u_{\varepsilon}^{n+1}\right\|_{V}^{p} \leqslant\|f\|_{\mathscr{V}^{\prime}}\left(\varepsilon \sum_{n=0}^{s}\left\|u_{\varepsilon}^{n+1}\right\|_{V}^{p}\right)^{1 / p}+(\Phi \circ i)^{*}\left(v^{0}\right)+\gamma, \\
& \forall s \in\{0, \ldots, N-1\} .
\end{aligned}
$$

We deduce, arguing by contradiction, that

$$
\exists C>0, \forall \varepsilon>0, \quad \forall s \in\{0, \ldots, N-1\}, \quad \varepsilon \sum_{n=0}^{s}\left\|u_{\varepsilon}^{n+1}\right\|_{V}^{p} \leqslant C .
$$

This estimation allows us to show that

$$
\exists C>0, \forall \varepsilon>0, \quad \forall s \in\{0, \ldots, N-1\}, \quad \varepsilon \sum_{n=0}^{s}\left\|A_{\varepsilon}^{n} u_{\varepsilon}^{n+1}\right\|_{V^{\prime}}^{q} \leqslant C .
$$

Indeed, from the first assertion of Lemma 2

$$
\begin{aligned}
\varepsilon \sum_{n=0}^{s}\left\|A_{\varepsilon}^{n} u_{\varepsilon}^{n+1}\right\|_{V^{\prime}}^{q} & \leqslant C \varepsilon \sum_{n=0}^{s}\left(1+\left\|u_{\varepsilon}^{n+1}\right\|_{V}^{p-1}\right)^{q} \\
& \leqslant C \varepsilon \sum_{n=0}^{s}\left(1+\left\|u_{\varepsilon}^{n+1}\right\|_{V}^{p}\right) \\
& \leqslant C .
\end{aligned}
$$

(We used the convexity inequality: $\forall r>0,(1+r)^{q} \leqslant 2^{q-1}\left(1+r^{q}\right)$ ).

Thus, we can bound the second term in (11), using (12) and (13):

$$
\begin{aligned}
\varepsilon\left|\sum_{n=0}^{s}\left\langle A_{\varepsilon}^{n} u_{\varepsilon}^{n+1}, u_{\varepsilon}^{n+1}\right\rangle\right| & \leqslant \varepsilon \sum_{n=0}^{s}\left\|A_{\varepsilon}^{n} u_{\varepsilon}^{n+1}\right\|_{V^{\prime}}\left\|u_{\varepsilon}^{n+1}\right\|_{V} \\
& \leqslant\left(\varepsilon \sum_{n=0}^{s}\left\|A_{\varepsilon}^{n} u_{\varepsilon}^{n+1}\right\|_{V^{\prime}}^{q}\right)^{1 / q}\left(\varepsilon \sum_{n=0}^{s}\left\|u_{\varepsilon}^{n+1}\right\|_{V}^{p}\right)^{1 / p} \\
& \leqslant C .
\end{aligned}
$$

Plugging into (11) this last result and the bound already obtained on the right-hand side term, we have

$$
\exists C>0, \forall \varepsilon>0, \forall s \in\{0, \ldots, N-1\}, \quad(\Phi \circ i)^{*}\left(v_{\varepsilon}^{s+1}\right) \leqslant C .
$$

On another hand, we deduce from (6) and (12)

$$
\exists C>0, \quad \forall \varepsilon>0, \quad \varepsilon \sum_{n=0}^{N-1}\left\|v_{\varepsilon}^{n+1}\right\|_{W^{\prime}}^{q} \leqslant C .
$$

At last, (13) and Lemma 1 applied to $\left(\mathrm{P}_{\varepsilon}\right)$ give

$$
\exists C>0, \quad \forall \varepsilon>0, \quad \varepsilon \sum_{n=0}^{N-1}\left\|\frac{v_{\varepsilon}^{n+1}-v_{\varepsilon}^{n}}{\varepsilon}\right\|_{V^{\prime}}^{q} \leqslant C .
$$




\subsection{Passing to the limit}

We denote by $u_{\varepsilon}$ the step function equal to $u_{\varepsilon}^{n+1}$ on $\left.] t_{n}, t_{n+1}\right]$ for $n=0, \ldots, N-1$, and $u^{0}$ for $t=0$. We define a function $v_{\varepsilon}$ in a similar way. We will also use the piecewise affine function $\widehat{v_{\varepsilon}}$ which coincides with $v_{\varepsilon}$ on points of the subdivision.

From the previous estimates, we deduce that there exists a subsequence (still indiced by $\varepsilon$ for sake of simplicity), such that

$$
\begin{aligned}
& u_{\varepsilon} \rightarrow u \text { weakly in } \mathscr{V} \text { from (12), } \\
& v_{\varepsilon} \rightarrow v \text { weakly in } \mathscr{W}^{\prime} \text { from (15). }
\end{aligned}
$$

We claim that the sequence $\widehat{v_{\varepsilon}}$ is convergent towards $v$. Indeed,

$$
\begin{aligned}
\left\|\widehat{v}_{\varepsilon}-v_{\varepsilon}\right\|_{\mathscr{W}^{\prime}}^{q} & =\int_{0}^{T}\left\|\widehat{v}_{\varepsilon}(t)-v_{\varepsilon}(t)\right\|_{W^{\prime}}^{q} \mathrm{~d} t \\
& =\sum_{n=0}^{N-1} \int_{t_{n}}^{t_{n+1}}\left\|v_{\varepsilon}^{n}+\frac{t-t_{n}}{\varepsilon}\left(v_{\varepsilon}^{n+1}-v_{\varepsilon}^{n}\right)-v_{\varepsilon}^{n+1}\right\|_{W^{\prime}}^{q} \mathrm{~d} t \\
& =\sum_{n=0}^{N-1}\left\|v_{\varepsilon}^{n+1}-v_{\varepsilon}^{n}\right\|_{W^{\prime}}^{q} \int_{t_{n}}^{t_{n+1}}\left|1-\frac{t-t_{n}}{\varepsilon}\right|^{q} \mathrm{~d} t \\
& =\frac{\varepsilon}{q+1} \sum_{n=0}^{N-1}\left\|v_{\varepsilon}^{n+1}-v_{\varepsilon}^{n}\right\|_{W^{\prime}}^{q} \\
& \leqslant C,
\end{aligned}
$$

using (15). Thus $\widehat{v_{\varepsilon}}$ is a bounded sequence of $\mathscr{W}^{\prime}$ and up to extract another subsequence we can assume that it converges weakly to some function of $\mathscr{W}^{\prime}$. But the same computations with the $\mathscr{V}^{\prime}$ norm lead to

$$
\left\|\widehat{v_{\varepsilon}}-v_{\varepsilon}\right\|_{\mathscr{V}^{\prime}}^{q} \leqslant C \varepsilon \sum_{n=0}^{N-1}\left\|v_{\varepsilon}^{n+1}-v_{\varepsilon}^{n}\right\|_{V^{\prime}}^{q} \leqslant C \varepsilon^{q} .
$$

from (16). Thus this limit has to be $v$ :

$$
\widehat{v_{\varepsilon}} \rightarrow v \text { weakly in } \mathscr{W}^{\prime} \text {. }
$$

\left.\left. Now the time derivative of ${\widehat{v_{\varepsilon}}}_{\text {on }}\right] t_{n}, t_{n+1}\right]$ is $\left(v_{\varepsilon}^{n+1}-v_{\varepsilon}^{n}\right) / \varepsilon$ so that from (16) we can derive

$$
\begin{aligned}
& \frac{\mathrm{d} \widehat{v}_{\varepsilon}}{\mathrm{d} t} \rightarrow \frac{\mathrm{d} v}{\mathrm{~d} t} \text { weakly in } \mathscr{V}^{\prime}, \\
& \mathscr{A}\left(u_{\varepsilon}\right) \rightarrow_{\chi} \text { weakly in } \mathscr{V}^{\prime}, \text { from (7). }
\end{aligned}
$$


We deduced weak convergences from our a priori estimates. We now mention a particular case of a compactness lemma from [20 ${ }^{1}$ which implies the strong convergence of a subsequence of $\left(v_{\varepsilon}\right)$ :

Lemma 4. If the sequence $\left(v_{\varepsilon}^{n}\right)_{0 \leqslant n \leqslant N}$ verifies (15) and (16), we can extract from $\left(v_{\varepsilon}\right)_{\varepsilon>0}$ a subsequence $\left(v_{\varepsilon^{\prime}}\right)_{\varepsilon^{\prime}>0}$ which strongly converges in $\mathscr{V}^{\prime}$ when $\varepsilon^{\prime}$ goes to 0.

To simplify we will write

$$
v_{\varepsilon}, \widehat{v_{\varepsilon}} \rightarrow v \text { strongly in } \mathscr{V}^{\prime} \text {. }
$$

Passing to the limit in the equation, we thus get in $\mathscr{V}^{\prime}$

$$
\frac{\mathrm{d} v}{\mathrm{~d} t}+\chi=f .
$$

We now have to show that $v \in \mathscr{B}(u), v(0)=v^{0}$ and $\chi=\mathscr{A}(u)$.

\subsection{End of the proof of Theorem 1 in the pseudo-monotone case}

- For the first statement, we write that for each couple $(y, x) \in \mathscr{V}^{\prime} \times \mathscr{V}$ such that $y \in \mathscr{B}(x)$ we have from the monotonicity of $\mathscr{B}$ :

$$
\left(v_{\varepsilon}-y, u_{\varepsilon}-x\right) \geqslant 0,
$$

and from (17) and (22) we have, passing to the limit

$$
(v-y, u-x) \geqslant 0 \text {. }
$$

As this procedure holds whatever couple $(y, x)$ we pick, we conclude from the maximality of $\mathscr{B}$ that $v \in \mathscr{B}(u)$.

- Turning to the initial condition, we observe that up to a redefinition of $\widehat{v_{\varepsilon}}$ and $v$ on a set of null measure, we can assume that they are absolutely continuous [9, p. 154]. On another hand (22) implies the existence of at least one $\left.t_{0} \in\right] 0, T[$ such that

$$
\widehat{v_{\varepsilon}}\left(t_{0}\right) \rightarrow v\left(t_{0}\right) \text { strongly in } V^{\prime} .
$$

Let $t \in[0, T]$, the absolute continuity of $\widehat{v_{\varepsilon}}$ allows us to write

$$
\widehat{v_{\varepsilon}}(t)=\widehat{v_{\varepsilon}}\left(t_{0}\right)+\int_{t_{0}}^{t} \frac{\mathrm{d} \widehat{v_{\varepsilon}}}{\mathrm{d} s} \mathrm{~d} s \rightarrow v\left(t_{0}\right)+\int_{t_{0}}^{t} \frac{\mathrm{d} v}{\mathrm{~d} s} \mathrm{~d} s \text { weakly in } V^{\prime},
$$

using (20). Thus

$$
\forall t \in[0, T], \quad \widehat{v_{\varepsilon}}(t) \rightarrow v(t) \text { weakly in } V^{\prime} .
$$

By definition, $\widehat{v}_{\varepsilon}(0)=v^{0}$, thus $v(0)=v^{0}$.

- In order to prove $\mathscr{A}(u)=\chi$, by a pseudo-monotonicity argument, we first have to prove that (2) holds i.e.

$$
\limsup _{\varepsilon \rightarrow 0}\left(\mathscr{A}\left(u_{\varepsilon}\right), u_{\varepsilon}-u\right) \leqslant 0 .
$$

\footnotetext{
${ }^{1}$ see also J.A. Dubinskii, Trans. AMS 1967, Weak convergence in nonlinear elliptic and parabolic equations.
} 
To this end, observe that (11) for $s=N-1$ becomes by definition of $A_{\varepsilon}^{n}$ and $f_{\varepsilon}^{n}$

$$
(\Phi \circ i)^{*}\left(v_{\varepsilon}(T)\right)+\left(\mathscr{A}\left(u_{\varepsilon}\right), u_{\varepsilon}\right) \leqslant\left(f, u_{\varepsilon}\right)+(\Phi \circ i)^{*}\left(v^{0}\right) .
$$

Using (21), we pass to the upper limit to get

$$
\limsup _{\varepsilon \rightarrow 0}\left(\mathscr{A}\left(u_{\varepsilon}\right), u_{\varepsilon}-u\right) \leqslant(f, u)+(\Phi \circ i)^{*}\left(v^{0}\right)-\liminf _{\varepsilon \rightarrow 0}(\Phi \circ i)^{*}\left(v_{\varepsilon}(T)\right)-(\chi, u) .
$$

As we proved that $v \in \mathscr{B}(u)$, taking $u$ as a test function in (23) and owing to a chain rule lemma adapted from the idea of Mignot [13] in the case of a Banach space (see $[10]$ or $[18,19]$ for a complete proof), we get

$$
(\chi, u)=(f, u)-(\Phi \circ i)^{*}(v(T))+(\Phi \circ i)^{*}\left(v^{0}\right) .
$$

Finally it remains to justify

$$
\liminf _{\varepsilon \rightarrow 0}(\Phi \circ i)^{*}\left(v_{\varepsilon}(T)\right) \geqslant(\Phi \circ i)^{*}(v(T)) .
$$

As $(\Phi \circ i)^{*}$ is convex and lower semi-continuous we only need for this to hold that

$$
v_{\varepsilon}(T) \rightarrow v(T) \text { weakly in } V^{\prime},
$$

which comes from (24) since $\widehat{v_{\varepsilon}}$ and $v_{\varepsilon}$ coincide for $t=T$.

If $\mathscr{A}$ is pseudo-monotone on $\mathscr{V},(2)$ gives $\chi=\mathscr{A}(u)$, and the existence proof if finished.

- Let us show now that for all time $t, v(t)$ remains in the domain of $(\Phi \circ i)^{*}$. For this, we point out that by construction,

$$
\left.\forall t \in] t_{n}, t_{n+1}\right], \quad \widehat{v_{\varepsilon}}(t) \in\left[v_{\varepsilon}^{n}, v_{\varepsilon}^{n+1}\right] .
$$

More explicitly,

$$
\widehat{v}_{\varepsilon}(t)=\left(1-\frac{t-t_{n}}{\varepsilon}\right) v_{\varepsilon}^{n}+\frac{t-t_{n}}{\varepsilon} v_{\varepsilon}^{n+1},
$$

so that the convexity of $(\Phi \circ i)^{*}$ implies

$$
(\Phi \circ i)^{*}\left(\widehat{v}_{\varepsilon}(t)\right) \leqslant\left(1-\frac{t-t_{n}}{\varepsilon}\right)(\Phi \circ i)^{*}\left(v_{\varepsilon}^{n}\right)+\frac{t-t_{n}}{\varepsilon}(\Phi \circ i)^{*}\left(v_{\varepsilon}^{n+1}\right) \leqslant C,
$$

from (14). Passing to the upper limit,

$$
\limsup _{\varepsilon \rightarrow 0}(\Phi \circ i)^{*}\left(\widehat{v}_{\varepsilon}(t)\right) \leqslant C,
$$

and a fortiori

$$
0 \leqslant(\Phi \circ i)^{*}(v(t)) \leqslant \liminf _{\varepsilon \rightarrow 0}(\Phi \circ i)^{*}\left(\widehat{v}_{\varepsilon}(t)\right) \leqslant C, \quad \forall t \in[0, T] .
$$

- At last let us show that $v \in L^{\infty}\left(0, T ; V^{\prime}\right)$. From the definition of $(\Phi \circ i)^{*}$ we have

$$
\forall u \in V, \quad(\Phi \circ i)^{*}\left(v_{\varepsilon}(t)\right) \geqslant\left\langle v_{\varepsilon}(t), u\right\rangle-(\Phi \circ i)(u) .
$$


Pick $\delta>0$ and $u=J^{-1} v_{\varepsilon}(t) / \delta\left\|v_{\varepsilon}(t)\right\|_{V^{\prime}}$, where $J$ is the duality operator ${ }^{2}$ from $V$ to $V^{\prime}$. We get

$$
\begin{gathered}
(\Phi \circ i)^{*}\left(v_{\varepsilon}(t)\right) \geqslant \frac{1}{\delta}\left\|v_{\varepsilon}(t)\right\|_{V^{\prime}}-\Phi \circ i\left(\frac{J^{-1} v_{\varepsilon}(t)}{\delta\left\|v_{\varepsilon}(t)\right\|_{V^{\prime}}}\right) \\
\geqslant \frac{1}{\delta}\left\|v_{\varepsilon}(t)\right\|_{V^{\prime}}-\sup _{\|u\|_{V}=1 / \delta} \Phi \circ i(u) \\
\left\|v_{\varepsilon}(t)\right\|_{V^{\prime}} \leqslant \delta(\Phi \circ i)^{*}\left(v_{\varepsilon}(t)\right)+\delta \sup _{\|u\| v=1 / \delta} \Phi \circ i(u) .
\end{gathered}
$$

Using (14), whose continuous counterpart is

$$
\exists C>0, \forall \varepsilon>0, \quad \sup _{t \in[0, T]} \operatorname{ess}(\Phi \circ i)^{*}\left(v_{\varepsilon}(t)\right) \leqslant C,
$$

in conjunction with assumption (6), and the convexity of $\Phi$ we have

$$
\exists C>0, \quad \forall \varepsilon>0, \quad \sup _{t \in[0, T]} \operatorname{ess}\left\|v_{\varepsilon}(t)\right\|_{V^{\prime}} \leqslant C .
$$

Moreover if we assume that $\Phi$ is continuous on $W$, then as $(\Phi \circ i)^{*}$ and $\Phi^{*}$ coincide on $W^{\prime}$ and $v_{\varepsilon}(t)$ belongs to $W^{\prime}$ for almost every $t$, the previous argument could be developed in the $\left\langle W^{\prime}, W\right\rangle$ duality and we get

$$
\exists C>0, \forall \varepsilon>0, \quad \sup _{t \in[0, T]} \operatorname{ess}\left\|v_{\varepsilon}(t)\right\|_{W^{\prime}} \leqslant C .
$$

\subsection{Compactness result}

If $\mathscr{A}$ is $\mathscr{B}$-pseudo-monotone, it remains to prove the strong convergence of a subsequence of $v_{\varepsilon}$ in $\mathscr{W}^{\prime}$. To this aim we state below a compactness result which could have its own interest in the study of doubly nonlinear equations.

Theorem 3. Let $V$ and $W$ be two separable and reflexive Banach spaces such that $V$ is densely and compactly embedded in $W$.

Let $E$ be a compact operator from $V$ to $W^{\prime}$. We define $\mathscr{E}: L^{p}(0, T ; V) \rightarrow L^{q}\left(0, T ; W^{\prime}\right)$ by

$$
\mathscr{E}(u)(t)=E(u(t)) \text { a.e. on }] 0, T[,
$$

and we assume that $\mathscr{E}$ is bounded (on the bounded sets of $L^{p}(0, T ; V)$ ).

Consider a bounded family $\left\{u_{\varepsilon}\right\}_{\varepsilon>0}$ in $L^{p}(0, T ; V)$, and denote by $\left\{v_{\varepsilon}\right\}_{\varepsilon>0} \subset$ $L^{q}\left(0, T ; W^{\prime}\right)$ the image family of $\left\{u_{\varepsilon}\right\}_{\varepsilon>0}$ by $\mathscr{E}$.

If moreover, $\left\{v_{\varepsilon}\right\}_{\varepsilon}>0$ verifies

$$
\lim _{h \rightarrow 0}\left\|\tau_{h} v_{\varepsilon}-v_{\varepsilon}\right\|_{L^{q}\left(0, T-h ; W^{\prime}\right)}=0 \quad \text { uniformly in } \varepsilon \text {, }
$$

then $\left\{v_{\varepsilon}\right\}_{\varepsilon>0}$ is relatively compact in $L^{q}\left(0, T ; W^{\prime}\right)$.

\footnotetext{
${ }^{2}$ We assume there that $V$ and $V^{\prime}$ are strictly convex (for $J$ to be mono-valued). If it was not the case we could re-norm them: see [27, II/B, p. 862].
} 
Remark 3. The case where $\mathscr{E}=0$ is straightforward. The case where $\mathscr{E}=I d$ is that of Simon [22, Theorem 3, p. 80], Theorem 3 is thus a nonlinear intermediate result.

Remark 4. We will use this compactness result with $E=B$. In the case where $\Phi$ has a strict convexity property, one could use results from Visintin [24] and their generalization by Balder and Valadier $[2,3]$.

Proof. Step 1: In order to show the compactness of $\left\{v_{\varepsilon}\right\}_{\varepsilon>0}$ in $L^{q}\left(0, T ; W^{\prime}\right)$, one only has to prove, owing to Theorem 1 of Simon [22] that for all $0<t_{1}<t_{2}<T, \int_{t_{1}}^{t_{2}} v_{\varepsilon}(t) \mathrm{d} t$ is relatively compact in $W^{\prime}$ (since we already have (29)).

Let us introduce the sets

$$
G_{\varepsilon}^{M}=\left\{t \in[0, T]:\left\|u_{\varepsilon}(t)\right\|_{V} \geqslant M\right\},
$$

and a constant $C>0$ such that

$$
\forall \varepsilon>0, \quad\left\|u_{\varepsilon}\right\|_{L^{p}(0, T ; V)} \leqslant C .
$$

Then meas $\left(G_{\varepsilon}^{h}\right) \leqslant C^{p} / M^{p}$. Set

$$
u_{\varepsilon}^{M}(t)= \begin{cases}u_{\varepsilon}(t) & \text { if } t \notin G_{\varepsilon}^{M}, \\ 0 & \text { elsewhere. }\end{cases}
$$

By construction,

$$
\forall M>0, \quad \forall \varepsilon>0, \quad \forall t \in[0, T], \quad\left\|u_{\varepsilon}^{M}(t)\right\|_{V} \leqslant M .
$$

As $E$ is compact from $V$ to $W^{\prime}$,

$$
\left\{v_{\varepsilon}^{M}(t)\right\}_{\varepsilon}=\left\{E\left(u_{\varepsilon}^{M}(t)\right)\right\}_{\varepsilon}
$$

is relatively compact in $W^{\prime}$ for all $M>0$.

Step 2: Let $\left.t_{1}, t_{2} \in\right] 0, T$ [ with $0<t_{1}<t_{2}<T$. For an integer $N$, we denote by $\left(s_{i}^{N}\right)_{0 \leqslant i \leqslant N}$ the subdivision of step $h=\left(t_{2}-t_{1}\right) / N$ of $\left[t_{1}, t_{2}\right]$.

Assume that for each $\eta>0$, there exist two integers $M_{1}$ and $N_{1}$ such that:

$$
\begin{aligned}
& \left.\forall M>M_{1}, \quad \forall N>N_{1}, \quad \forall \varepsilon>0, \quad \exists s_{\varepsilon} \in\right] 0, h[, \\
& \left\|\int_{t_{1}}^{t_{2}} v_{\varepsilon}(t) \mathrm{d} t-\int_{t_{1}}^{t_{2}} \sum_{i=1}^{N} v_{\varepsilon}^{M}\left(s_{i-1}^{N}+s_{\varepsilon}\right) \chi_{] s_{i-1}^{N}, s_{i}^{N}\right]}(t) \mathrm{d} t\right\|_{W^{\prime}}<\eta .
\end{aligned}
$$

From step 1, for fixed $M, N$ the sequence in $\varepsilon$

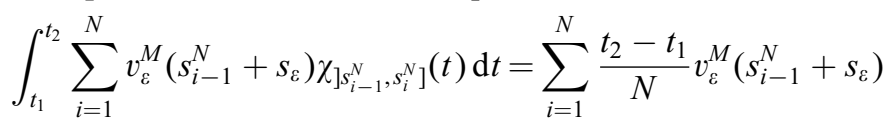

is relatively compact in $W^{\prime}$. Thus owing to the convergence when $M, N \rightarrow \infty$, uniform in $\varepsilon$, stated in (30) the sequence

$$
\left\{\int_{t_{1}}^{t_{2}} v_{\varepsilon}(t) \mathrm{d} t\right\}_{\varepsilon>0}
$$

is relatively compact in $W^{\prime}$, as uniform limit of relatively compact subset, and the proof of theorem ends up. 
Step 3: To prove (30), we argue by contradiction, assuming that such a family $\left\{s_{\varepsilon}\right\}$ does not exist; that means there exists $\eta>0$, such that for all $M_{1}>0$ and $N_{1}>0$,

$$
\begin{aligned}
& \left.\exists M>M_{1}, \quad \exists N>N_{1}, \quad \exists \varepsilon>0, \forall s \in\right] 0, h[ \\
& \quad\left\|\int_{t_{1}}^{t_{2}}\left(v_{\varepsilon}(t)-\sum_{i=1}^{N} v_{\varepsilon}^{M}\left(s_{i-1}^{N}+s\right) \chi_{] s_{i-1}^{N}, s_{i}^{N}\right]}(t)\right) \mathrm{d} t\right\|_{W^{\prime}} \geqslant \eta .
\end{aligned}
$$

Then a fortiori

$$
\forall s \in] 0, h\left[, \quad \int_{t_{1}}^{t_{2}}\left\|v_{\varepsilon}(t)-\sum_{i=1}^{N} v_{\varepsilon}^{M}\left(s_{i-1}^{N}+s\right) \chi_{] s_{i-1}^{N}, s_{i}^{N}\right]}(t)\right\|_{W^{\prime}} \mathrm{d} t \geqslant \eta,\right.
$$

and integrating with respect to $s$ from 0 to $h=\left(t_{2}-t_{1}\right) / N$ we have

$$
\begin{aligned}
& \exists M>M_{1}, \quad \exists N>N_{1}, \exists \varepsilon>0, \\
& \frac{1}{h} \int_{0}^{h} \int_{t_{1}}^{t_{2}}\left\|v_{\varepsilon}(t)-\sum_{i=1}^{N} v_{\varepsilon}^{M}\left(s_{i-1}^{N}+s\right) \chi_{] s_{i-1}^{N}, s_{i}^{N}\right]}(t)\right\|_{W^{\prime}} \mathrm{d} t \mathrm{~d} s \geqslant \eta .
\end{aligned}
$$

Let us show that this relation leads to a contradiction. Indeed,

$$
\begin{aligned}
& \frac{1}{h} \int_{0}^{h} \int_{t_{1}}^{t_{2}}\left\|v_{\varepsilon}(t)-\sum_{i=1}^{N} v_{\varepsilon}^{M}\left(s_{i-1}^{N}+s\right) \chi_{]_{s_{i-1}}^{N}, s_{i}^{N}\right]}(t)\right\|_{W^{\prime}} \mathrm{d} t \mathrm{~d} s \\
& =\frac{1}{h} \int_{0}^{h} \sum_{i=1}^{N} \int_{s_{i-1}^{N}}^{s_{i}^{N}}\left\|v_{\varepsilon}(t)-v_{\varepsilon}^{M}\left(s_{i-1}^{N}+s\right)\right\|_{W^{\prime}} \mathrm{d} t \mathrm{~d} s \\
& =\frac{1}{h} \sum_{i=1}^{N} \int_{s_{i-1}^{N}}^{s_{i}^{N}} \int_{s_{i-1}^{N}}^{s_{i}^{N}}\left\|v_{\varepsilon}(t)-v_{\varepsilon}^{M}(s)\right\|_{W^{\prime}} \mathrm{d} t \mathrm{~d} s
\end{aligned}
$$

and by Fubini's theorem, setting $\sigma=s-t$,

$$
\begin{aligned}
& =\frac{1}{h} \sum_{i=1}^{N} \int_{s_{i-1}^{N}}^{s_{i}^{N}} \int_{s_{i-1}^{N}-t}^{s_{i}^{N}-t}\left\|v_{\varepsilon}(t)-v_{\varepsilon}^{M}(t+\sigma)\right\|_{W^{\prime}} \mathrm{d} \sigma \mathrm{d} t \\
& =\frac{1}{h} \int_{-h}^{h} \sum_{i=1}^{N} \int_{\max \left(s_{i-1}^{N}, s_{i-1}^{N}-\sigma\right)}^{\min \left(s_{i}^{N}, s_{i}^{N}-\sigma\right)}\left\|v_{\varepsilon}(t)-v_{\varepsilon}^{M}(t+\sigma)\right\|_{W^{\prime}} \mathrm{d} t \mathrm{~d} \sigma \\
& \leqslant \frac{1}{h} \int_{-h}^{h} \int_{\max \left(t_{1}, t_{1}-\sigma\right)}^{\min \left(t_{2}, t_{2}-\sigma\right)}\left\|v_{\varepsilon}(t)-v_{\varepsilon}^{M}(t+\sigma)\right\|_{W^{\prime}} \mathrm{d} t \mathrm{~d} \sigma
\end{aligned}
$$




$$
\begin{aligned}
\leqslant & \frac{1}{h} \int_{-h}^{h} \int_{\max \left(t_{1}, t_{1}-\sigma\right)}^{\min \left(t_{2}, t_{2}-\sigma\right)}\left\|v_{\varepsilon}(t)-v_{\varepsilon}(t+\sigma)\right\|_{W^{\prime}} \mathrm{d} t \mathrm{~d} \sigma \\
& +\frac{1}{h} \int_{-h}^{h} \int_{\max \left(t_{1}, t_{1}-\sigma\right)}^{\min \left(t_{2}, t_{2}-\sigma\right)} \chi_{G_{\varepsilon}^{M}}(t+\sigma)\left\|v_{\varepsilon}(t)\right\|_{W^{\prime}} \mathrm{d} t \mathrm{~d} \sigma \\
\leqslant & 2 T^{1-1 / p} \sup _{\sigma \in[-h, h]}\left(\int_{0}^{T-\sigma}\left\|v_{\varepsilon}(t)-v_{\varepsilon}(t+\sigma)\right\|_{W^{\prime}}^{q} \mathrm{~d} t\right)^{1 / q}+2 \frac{C}{M}\left\|v_{\varepsilon}\right\|_{L^{q}\left(0, T ; W^{\prime}\right) .}
\end{aligned}
$$

Let $\eta>0$ be fixed.

Owing to (29), there exists $N_{1}>0$ such that for any $N>N_{1}$, with the subdivision of step $h=\left(t_{2}-t_{1}\right) / N$ we have

$$
\forall \varepsilon>0, \quad \sup _{\sigma \in[-h, h]}\left(\int_{0}^{T-\sigma}\left\|v_{\varepsilon}(t)-v_{\varepsilon}(t+\sigma)\right\|_{W^{\prime}}^{q} \mathrm{~d} t\right)^{1 / q} \leqslant \frac{\eta}{4 T^{1 / p}} .
$$

On another hand, $\mathscr{E}$ has been assumed to be bounded on bounded sets, and $\left\|u_{\varepsilon}\right\|_{L^{p}(0, T ; V)}$ being bounded by assumption, this is still true for $\left\|v_{\varepsilon}\right\|_{L^{q}\left(0, T ; W^{\prime}\right)}$; thus there exists $M_{1}>0$, such that:

$$
\forall M>M_{1}, \quad \forall \varepsilon>0, \quad \frac{C}{M}\left\|v_{\varepsilon}\right\|_{L^{q}\left(0, T ; W^{\prime}\right)} \leqslant \frac{\eta}{4} .
$$

Gathering the last two inequalities, we derive a contradiction with (32), which ends the proof of Theorem 3 .

\subsection{End of the proof of Theorem 1}

Let us recall that it remains to study the passing to the limit in nonlinear terms of $\mathscr{A}$, using a strong convergence of $v_{\varepsilon}$ thanks to the previous compactness lemma. Let us start by proving that the sequence $v_{\varepsilon}$ defined in 4.3 verifies (29), postponing the proof of the following lemma.

Lemma 5. Assume $B$ is continuous from $W$ to $W^{\prime}$. Then the conditions:

(i) $u_{\varepsilon} \rightarrow u$ weakly in $\mathscr{V}$,

(ii) $\exists C>0, \forall \varepsilon>0, \forall h \in] 0, T[$,

$$
\int_{0}^{T-h}\left\langle v_{\varepsilon}(t+h)-v_{\varepsilon}(t), u_{\varepsilon}(t+h)-u_{\varepsilon}(t)\right\rangle_{W^{\prime}, W} \mathrm{~d} t \leqslant C h^{1 / p},
$$

(iii) $\exists C>0, \forall \varepsilon>0$, $\sup _{t \in[0, T]} \operatorname{ess}\left\|v_{\varepsilon}(t)\right\|_{W^{\prime}} \leqslant C$.

imply (29).

We already have (i) from (17), and (iii) Owing to (28) (Remember that in the $\mathscr{B}$-pseudo-monotone case we assumed that $\mathscr{B}$ is continuous, and so is $\Phi$ ).

To establish (ii), we point out that we only have to consider the case where $h=k \varepsilon, k \in\{1, \ldots, N-1\}$, since $v_{\varepsilon}$ and $u_{\varepsilon}$ are step functions. Indeed, assume that this 
inequality holds for $k \varepsilon$. Let $h \in] k \varepsilon,(k+1) \varepsilon\left[\right.$, with $k \geqslant 1$, and $h_{0}=h-k \varepsilon$.

$$
\begin{aligned}
\int_{0}^{T-h} & \left\langle v_{\varepsilon}(t+h)-v_{\varepsilon}(t), u_{\varepsilon}(t+h)-u_{\varepsilon}(t)\right\rangle \mathrm{d} t \\
= & \left(\varepsilon-h_{0}\right) \sum_{n=1}^{N-k}\left\langle v_{\varepsilon}^{n+k}-v_{\varepsilon}^{n}, u_{\varepsilon}^{n+k}-u_{\varepsilon}^{n}\right\rangle+h_{0} \sum_{n=1}^{N-k-1}\left\langle v_{\varepsilon}^{n+k+1}-v_{\varepsilon}^{n}, u_{\varepsilon}^{n+k+1}-u_{\varepsilon}^{n}\right\rangle \\
= & \frac{\varepsilon-h_{0}}{\varepsilon} \sum_{n=1}^{N-k} \int_{t_{n-1}}^{t_{n}}\left\langle v_{\varepsilon}(t+k \varepsilon)-v_{\varepsilon}(t), u_{\varepsilon}(t+k \varepsilon)-u_{\varepsilon}(t)\right\rangle \mathrm{d} t \\
& +\frac{h_{0}}{\varepsilon} \sum_{n=1}^{N-k-1} \int_{t_{n-1}}^{t_{n}}\left\langle v_{\varepsilon}(t+(k+1) \varepsilon)-v_{\varepsilon}(t), u_{\varepsilon}(t+(k+1) \varepsilon)-u_{\varepsilon}(t)\right\rangle \mathrm{d} t .
\end{aligned}
$$

We thus have

$$
\begin{aligned}
\int_{0}^{T-h} & \left\langle v_{\varepsilon}(t+h)-v_{\varepsilon}(t), u_{\varepsilon}(t+h)-u_{\varepsilon}(t)\right\rangle \mathrm{d} t \\
= & \frac{\varepsilon-h_{0}}{\varepsilon} \int_{0}^{T-k \varepsilon}\left\langle v_{\varepsilon}(t+k \varepsilon)-v_{\varepsilon}(t), u_{\varepsilon}(t+k \varepsilon)-u_{\varepsilon}(t)\right\rangle \mathrm{d} t \\
& +\frac{h_{0}}{\varepsilon} \int_{0}^{T-(k+1) \varepsilon}\left\langle v_{\varepsilon}(t+(k+1) \varepsilon)-v_{\varepsilon}(t), u_{\varepsilon}(t+(k+1) \varepsilon)-u_{\varepsilon}(t)\right\rangle \mathrm{d} t \\
\leqslant & C\left[\frac{\varepsilon-h_{0}}{\varepsilon}(k \varepsilon)^{1 / p}+\frac{h_{0}}{\varepsilon}((k+1) \varepsilon)^{1 / p}\right] \\
\leqslant & C\left[\frac{\left(\varepsilon-h_{0}\right) k \varepsilon+h_{0}(k+1) \varepsilon}{\varepsilon}\right]^{1 / p} \leqslant C h^{1 / p},
\end{aligned}
$$

using the concavity of $r \rightarrow r^{1 / p}$.

It remains to prove that $\exists C>0, \forall \varepsilon>0, \forall k \in\{1, \ldots, N-1\}$,

$$
\int_{0}^{T-k \varepsilon}\left\langle v_{\varepsilon}(t+k \varepsilon)-v_{\varepsilon}(t), u_{\varepsilon}(t+k \varepsilon)-u_{\varepsilon}(t)\right\rangle \mathrm{d} t \leqslant C(k \varepsilon)^{1 / p}
$$

which rewritten in discrete quantities means:

$$
\varepsilon \sum_{n=0}^{N-k-1}\left\langle v_{\varepsilon}^{n+k+1}-v_{\varepsilon}^{n+1}, u_{\varepsilon}^{n+k+1}-u_{\varepsilon}^{n+1}\right\rangle \leqslant C(k \varepsilon)^{1 / p} .
$$

In order to show (33) we write the approximated problem under variational form:

Find $\left(u_{\varepsilon}^{n}\right)_{n=0, \ldots, N} \in V^{N+1}$ such that

$$
\varepsilon \sum_{n=0}^{N-1}\left\langle\frac{v_{\varepsilon}^{n+1}-v_{\varepsilon}^{n}}{\varepsilon}, w_{n}\right\rangle+\varepsilon \sum_{n=0}^{N-1}\left\langle A_{\varepsilon}^{n} u_{\varepsilon}^{n+1}, w_{n}\right\rangle=\varepsilon \sum_{n=0}^{N-1}\left\langle f_{\varepsilon}^{n}, w_{n}\right\rangle \quad \forall w=\left(w_{n}\right) \in V^{N+1} .
$$


Let $k \in\{0, \ldots, N-1\}$ and $m \in\{0, \ldots, N-k-1\}$ be fixed. We choose as a test function the $(N+1)$-uple $w$ those components are $u_{\varepsilon}^{m+k+1}-u_{\varepsilon}^{m+1}$ for all $n \in\{m+1, \ldots, m+k\}$ and zero for other values of $n$. By construction,

$$
\varepsilon \sum_{n=0}^{N-1}\left\langle\frac{v_{\varepsilon}^{n+1}-v_{\varepsilon}^{n}}{\varepsilon}, w_{n}\right\rangle=\left\langle v_{\varepsilon}^{m+k+1}-v_{\varepsilon}^{m}, u_{\varepsilon}^{m+k+1}-u_{\varepsilon}^{m+1}\right\rangle .
$$

We have to bound from above the sum on $m$ of these quantities to get (33). Let us compute:

$$
\begin{aligned}
\varepsilon \sum_{n=0}^{N-1}\left\langle f_{\varepsilon}^{n}-A_{\varepsilon}^{n} u_{\varepsilon}^{n+1}, w_{n}\right\rangle & \\
= & \varepsilon\left\langle\sum_{n=m+1}^{m+k} f_{\varepsilon}^{n}-A_{\varepsilon}^{n} u_{\varepsilon}^{n+1}, u_{\varepsilon}^{m+k+1}-u_{\varepsilon}^{m+1}\right\rangle \\
\leqslant & \varepsilon\left(\sum_{n=m+1}^{m+k}\left\|f_{\varepsilon}^{n}\right\|_{V^{\prime}}+\left\|A_{\varepsilon}^{n} u_{\varepsilon}^{n+1}\right\|_{V^{\prime}}\right)\left\|u_{\varepsilon}^{m+k+1}-u_{\varepsilon}^{m+1}\right\|_{V} \\
\leqslant & \left(\varepsilon \sum_{n=m+1}^{m+k} 1^{p}\right)^{1 / p}\left[\left(\varepsilon \sum_{n=0}^{N-1}\left\|f_{\varepsilon}^{n}\right\|_{V^{\prime}}^{q}\right)^{1 / q}\right. \\
& \left.+\left(\varepsilon \sum_{n=0}^{N-1}\left\|A_{\varepsilon}^{n} u_{\varepsilon}^{n+1}\right\|_{V^{\prime}}^{q}\right)^{1 / q}\right]\left\|_{\varepsilon}^{m+k+1}-u_{\varepsilon}^{m+1}\right\|_{V} \\
\leqslant & (k \varepsilon)^{1 / p}\left(\left\|f_{\varepsilon}\right\|_{\mathscr{V}^{\prime}}+C\right)\left(\left\|u_{\varepsilon}^{m+k+1}\right\|_{V}+\left\|u_{\varepsilon}^{m+1}\right\|_{V}\right) \quad \text { from (13) } \\
\leqslant & C(k \varepsilon)^{1 / p}\left(\left\|u_{\varepsilon}^{m+k+1}\right\|_{V}+\left\|u_{\varepsilon}^{m+1}\right\|_{V}\right) .
\end{aligned}
$$

This leads for the quantity we are willing to estimate:

$$
\begin{aligned}
& \varepsilon \sum_{m=0}^{N-k-1}\left\langle v_{\varepsilon}^{m+k+1}-v_{\varepsilon}^{m}, u_{\varepsilon}^{m+k+1}-u_{\varepsilon}^{m+1}\right\rangle \\
& \quad \leqslant C(k \varepsilon)^{1 / p} \varepsilon \sum_{m=0}^{N-k-1}\left(\left\|u_{\varepsilon}^{m+k+1}\right\|_{V}+\left\|u_{\varepsilon}^{m+1}\right\|_{V}\right) \\
& \quad \leqslant C(k \varepsilon)^{1 / p}((N-k) \varepsilon)^{1 / q}\left\|u_{\varepsilon}\right\|_{\mathscr{V}} \\
& \quad \leqslant C(k \varepsilon)^{1 / p}(T)^{1 / q} \\
& \quad \leqslant C(k \varepsilon)^{1 / p} .
\end{aligned}
$$


Thus we have (ii), then (29) if we admit Lemma 5. Applying Theorem 3, we get the strong convergence of a subsequence of $v_{\varepsilon}$, and we conclude the proof of Theorem 1 by the definition of $\mathscr{B}$-pseudo-monotonicity. We refer to annex for the demonstration of Lemma 5 which is purely technical.

\subsection{Application: a class of $\mathscr{B}$-pseudo-monotone operators}

Let $N, p, r$ be three integers with $N>0, p>r>1$. The conjugate exponents of $p$ and $r$ will be denoted by $q$ and $s$, respectively:

$$
\frac{1}{p}+\frac{1}{q}=1, \quad \frac{1}{r}+\frac{1}{s}=1 .
$$

Consider an open set $\Omega$ of $\mathbb{R}^{N}$, and $\left.Q=\Omega \times\right] 0, T[$.

We denote by $\Gamma_{1}$ some part of $\left.\partial \Omega, \Sigma_{1}=\Gamma_{1} \times\right] 0, T\left[\right.$, and by $\left.\Sigma_{2}=\left(\partial \Omega \backslash \Gamma_{1}\right) \times\right] 0, T[$.

Let $V$ be the closed subspace of $W^{1, p}(\Omega)$, containing $W_{0}^{1, p}(\Omega)$, and defined by

$$
V=\left\{u \in W^{1, p}(\Omega), u_{\mid \Gamma_{1}}=0\right\} .
$$

We set

$$
\Phi(u)=\int_{\Omega} g(x, u(x)) \mathrm{d} x
$$

where $g$ is a measurable function on $\Omega \times \mathbf{R}$ such that $\forall x \in \Omega, r \rightarrow g(x, r)$ is proper lower semi-continuous and convex on R. $g$ is a normal convex integrand, in the sense of Rockafellar (cf. [21, Proposition 1, p. 221]).

For any measurable function $u$ on $\Omega, x \rightarrow g(x, u(x))$ is measurable on $\Omega$ and $\Phi$ is proper, lower semi-continuous and convex on $W$.

Denoting by $\beta: \Omega \times \mathbf{R} \rightarrow \mathbf{R}$ the sub-differential of $g(x,$.$) we have for u \in W$

$$
\partial \Phi(u)(x)=\beta(x, u(x)) \quad \text { a.e. on } \Omega .
$$

We moreover assume that $z \mapsto \beta(x, z)$ is continuous and strictly increasing for almost every $x \in \Omega$, and verifies the following growth assumption:

$$
\exists a_{1}, a_{2}>0:|\beta(x, z)| \leqslant a_{1}|z|^{p-1}+a_{2}, \quad \forall z \in \mathbb{R}, \text { a.e. on } \Omega \text {. }
$$

Then operator $B$ associated to $\beta$ verifies assumptions (5) and (6), and $\mathscr{B}$ is continuous from $L^{p}(Q)$ to $L^{q}(Q)$.

For the elliptic part, we introduce a family of real functions $A_{i}(x, t, \eta, v, \xi), i \in$ $\{0, \ldots, N\}$ defined on $Q \times \mathbb{R} \times \mathbb{R} \times \mathbb{R}^{N}$ and verifying:

(A1) For almost all $(x, t) \in Q$, the function $(\eta, v, \xi) \mapsto A_{i}(x, t, \eta, v, \xi)$ is continuous on $\mathbb{R} \times \mathbb{R} \times \mathbb{R}^{N}$ and for all $(\eta, v, \xi)$ the function $(x, t) \mapsto A_{i}(x, t, \eta, v, \xi)$ is measurable on $Q$.

(A2) For all $(u, v, w)$ belonging to $L^{r}(Q) \times L^{q}(Q) \times\left(L^{p}(Q)\right)^{N}$,

$$
(x, t) \rightarrow A_{i}(x, t, u(x, t), v(x, t), w(x, t))
$$

belongs to $L^{q}(Q)$ for $i \in\{1, \ldots, N\}$ and to $L^{s}(Q)$ for $i=0$.

The vector valued function $\mathbf{A}=\left(A_{i}\right)_{1 \leqslant i \leqslant N}$ verifies, 
(A3) For fixed $x, t$ a.e. in $Q$ and bounded $|\eta|$,

$$
\lim _{|\xi| \rightarrow+\infty} \frac{\mathbf{A}(x, t, \eta, \beta(x, \eta), \xi) \cdot \xi}{|\xi|+|\xi|^{p-1}}=+\infty
$$

(A4) Almost everywhere in $Q$ and for any $\eta$,

$$
\left(\mathbf{A}(x, t, \eta, \beta(x, \eta), \xi)-\mathbf{A}\left(x, t, \eta, \beta(x, \eta), \xi^{*}\right)\right) \cdot\left(\xi-\xi^{*}\right)>0 \quad \text { if } \xi \neq \xi^{*} .
$$

The operator $\mathscr{A}: L^{p}(0, T ; V) \mapsto L^{q}\left(0, T ; V^{\prime}\right)$ is then defined for $u \in L^{p}(0, T ; V)$ by

$$
\begin{aligned}
(\mathscr{A}(u), v)= & \int_{0}^{T} \int_{\Omega} \mathbf{A}(x, t, u(x, t), \beta(x, u(x, t)), \nabla u(x, t)) \cdot \nabla v(x, t) \mathrm{d} x \mathrm{~d} t \\
& +\int_{0}^{T} \int_{\Omega} A_{0}(x, t, u(x, t), \beta(x, u(x, t)), \nabla u(x, t)) v(x, t) \mathrm{d} x \mathrm{~d} t,
\end{aligned}
$$

for all $v \in L^{p}(0, T ; V)$.

Proposition 2. The operator $\mathscr{A}$ is $\mathscr{B}$-pseudo-monotone on $L^{p}(0, T ; V)$.

Proof. We adapt the proof of Lions ([6], pages 182-185), considering a sequence $u_{n}$ such that

(i) $u_{n} \rightarrow u$ weakly in $L^{p}(0, T ; V)$.

(ii) $\mathscr{B}\left(u_{n}\right) \rightarrow \mathscr{B}(u)$ strongly in $L^{q}(Q)$.

(iii) $\limsup _{n \rightarrow \infty}\left(\mathscr{A}\left(u_{n}\right), u_{n}-u\right) \leqslant 0$.

Assumption (ii) implies up to a subsequence that

$$
\beta\left(x, u_{n}(x, t)\right) \rightarrow \beta(x, u(x, t)) \text { a.e. on } Q .
$$

As $\beta$ is strictly increasing and continuous we have

$$
u_{n}(x, t) \rightarrow u(x, t) \quad \text { a.e. on } Q .
$$

We deduce from this, (i) and $r<p$ that

$$
u_{n} \rightarrow u \quad \text { strong in } L^{r}(Q) \text {. }
$$

For sake of readability, we denote by $A_{i}(u, \mathscr{B}(u), \nabla u)$ the function of $L^{q}(Q)$ equal to

$$
A_{i}(x, t, u(x, t), \mathscr{B}(u)(x, t), \nabla u(x, t)) \quad \text { almost everywhere on } Q
$$

and this value will be abbreviated by $A_{i}(x, t, u, \mathscr{B}(u), \nabla u)$. The symbol (, ) still denotes the duality product between $\mathscr{V}^{\prime}$ and $\mathscr{V}$ or between $L^{q}(Q)^{N}$ and $L^{p}(Q)^{N}$, and the dot (.) represents the scalar product of $\mathbb{R}^{N}$.

We have

$$
\begin{aligned}
\left(\mathscr{A}\left(u_{n}\right), u_{n}-u\right)= & \left(\mathbf{A}\left(u_{n}, \mathscr{B}\left(u_{n}\right), \nabla u_{n}\right), \nabla\left(u_{n}-u\right)\right)+\left(A_{0}\left(u_{n}, \mathscr{B}\left(u_{n}\right), \nabla u_{n}\right), u_{n}-u\right) \\
= & \left(\mathbf{A}\left(u_{n}, \mathscr{B}\left(u_{n}\right), \nabla u_{n}\right)-\mathbf{A}\left(u_{n}, \mathscr{B}\left(u_{n}\right), \nabla u\right), \nabla\left(u_{n}-u\right)\right) \\
& +\left(\mathbf{A}\left(u_{n}, \mathscr{B}\left(u_{n}\right), \nabla u\right), \nabla\left(u_{n}-u\right)\right) \\
& +\left(A_{0}\left(u_{n}, \mathscr{B}\left(u_{n}\right), \nabla u_{n}\right), u_{n}-u\right) .
\end{aligned}
$$


Step 1: Let us show that we can extract a subsequence such that

$$
\left(\mathbf{A}\left(u_{n}, \mathscr{B}\left(u_{n}\right), \nabla u_{n}\right)-\mathbf{A}\left(u_{n}, \mathscr{B}\left(u_{n}\right), \nabla u\right), \nabla\left(u_{n}-u\right)\right) \rightarrow 0 .
$$

Assumptions (A1) and (A2) imply [23, Theorem 19.1, p. 154, and p. 162] that the application

$$
(u, v, w) \rightarrow A_{i}(u, v, w)
$$

is continuous and bounded (on bounded sets) from $L^{r}(Q) \times L^{q}(Q) \times\left(L^{p}(Q)\right)^{N}$ to $L^{q}(Q)$ for $i>0$ and to $L^{S}(Q)$ for $i=0 .^{3}$

We deduce that $\mathbf{A}\left(u_{n}, \mathscr{B}\left(u_{n}\right), \nabla u_{n}\right)$ remains in a bounded set of $\left(L^{q}(Q)\right)^{N}$, and that

$$
\mathbf{A}\left(u_{n}, \mathscr{B}\left(u_{n}\right), \nabla u\right) \rightarrow \mathbf{A}(u, \mathscr{B}(u), \nabla u) \quad \text { strongly in }\left(L^{q}(Q)\right)^{N} .
$$

This strong convergence and (i) imply

$$
\left(\mathbf{A}\left(u_{n}, \mathscr{B}\left(u_{n}\right), \nabla u\right), \nabla\left(u_{n}-u\right)\right) \rightarrow 0 .
$$

On another hand, we know that $A_{0}$ is bounded from $L^{r}(Q) \times L^{q}(Q) \times\left(L^{p}(Q)\right)^{N}$ to $L^{S}(Q)$, which allows us to write

$$
\left|\left(A_{0}\left(u_{n}, \mathscr{B}\left(u_{n}\right), \nabla u_{n}\right), u_{n}-u\right)\right| \leqslant C\left\|u_{n}-u\right\|_{L^{r}(Q)} \rightarrow 0 .
$$

Using (iii), (41) and (42) in (38) we get

$$
\limsup _{n \rightarrow \infty}\left(\mathbf{A}\left(u_{n}, \mathscr{B}\left(u_{n}\right), \nabla u_{n}\right)-\mathbf{A}\left(u_{n}, \mathscr{B}\left(u_{n}\right), \nabla u\right), \nabla\left(u_{n}-u\right)\right) \leqslant 0,
$$

and thanks to (A4) we have (39).

Step 2: Let us show that this condition (39) implies

$$
\mathbf{A}\left(u_{n}, \mathscr{B}\left(u_{n}\right), \nabla u_{n}\right) \rightarrow \mathbf{A}(u, \mathscr{B}(u), \nabla u) \quad \text { weakly in }\left(L^{q}(Q)\right)^{N}
$$

and

$$
A_{0}\left(u_{n}, \mathscr{B}\left(u_{n}\right), \nabla u_{n}\right) \rightarrow A_{0}(u, \mathscr{B}(u), \nabla u) \text { weakly in } L^{s}(Q) .
$$

Indeed, denoting by

$$
F_{n}(x, t)=\left(\mathbf{A}\left(x, t, u_{n}, \mathscr{B}\left(u_{n}\right), \nabla u_{n}\right)-\mathbf{A}\left(x, t, u_{n}, \mathscr{B}\left(u_{n}\right), \nabla u\right)\right) \cdot \nabla\left(u_{n}-u\right) \geqslant 0,
$$

we have $\int_{Q} F_{n}(x, t) \mathrm{d} x \mathrm{~d} t \rightarrow 0$ from (39), thus there exists a subset $Z \subset Q$ of null measure, such that up to a subsequence,

$$
u_{n}(x, t) \rightarrow u(x, t), \quad \mathscr{B}\left(u_{n}\right)(x, t) \rightarrow \mathscr{B}(u)(x, t), \quad F_{n}(x, t) \rightarrow 0, \quad \forall(x, t) \in Q \backslash Z .
$$

Let $(x, t) \notin Z$, and $\xi^{*}(x, t)$ a limit of $\nabla u_{n}(x, t)$. We surely have $\left|\xi^{*}(x, t)\right|<+\infty$. Otherwise we would have from (A3)

$$
\frac{\mathbf{A}\left(x, t, u_{n}, \mathscr{B}\left(u_{n}\right), \nabla u_{n}\right) \cdot \nabla u_{n}(x, t)}{\left|\nabla u_{n}(x, t)\right|+\left|\nabla u_{n}(x, t)\right|^{p-1}} \rightarrow+\infty,
$$

and then

$$
\left\|F_{n}\right\|_{L^{1}(Q)} \rightarrow+\infty
$$

\footnotetext{
${ }^{3}$ See also [15].
} 
which leads to a contradiction. To the limit we have from (A1),

$$
\left(\mathbf{A}\left(x, t, u, \mathscr{B}(u), \xi^{*}\right)-\mathbf{A}(x, t, u, \mathscr{B}(u), \nabla u)\right) \cdot\left(\xi^{*}(x, t)-\nabla u(x, t)\right)=0,
$$

almost everywhere on $Q$, which means from (A4) that $\xi^{*}(x, t)=\nabla u(x, t)$. We showed that

$$
\forall i \in\{0, \ldots, N\}, \quad A_{i}\left(x, t, u_{n}, \mathscr{B}\left(u_{n}\right), \nabla u_{n}\right) \rightarrow \mathbf{A}(x, t, u, \mathscr{B}(u), \nabla u) \quad \text { a.e. on } Q .
$$

As operators $A_{i}$ are bounded on $L^{q}(Q)$ and $L^{s}(Q)$ we obtain (43) and (44) (see for instance [16, p. 12]).

Step 3: Let $w=(1-\theta) u+\theta v, \theta \in] 0,1[$, we have from (A4)

$$
\left(\mathbf{A}\left(u_{n}, \mathscr{B}\left(u_{n}\right), \nabla u_{n}\right)-\mathbf{A}\left(u_{n}, \mathscr{B}\left(u_{n}\right), \nabla w\right), \nabla\left(u_{n}-w\right)\right) \geqslant 0 \quad \forall w .
$$

Consequently,

$$
\begin{aligned}
\theta\left(\mathbf{A}\left(u_{n}, \mathscr{B}\left(u_{n}\right), \nabla u_{n}\right), \nabla\left(u_{n}-v\right)\right) \geqslant & -(1-\theta)\left(\mathbf{A}\left(u_{n}, \mathscr{B}\left(u_{n}\right), \nabla u_{n}\right), \nabla\left(u_{n}-u\right)\right) \\
& +\left(\mathbf{A}\left(u_{n}, \mathscr{B}\left(u_{n}\right), \nabla w\right), \nabla\left(u_{n}-w\right)\right) .
\end{aligned}
$$

The first term of the right hand side of (45) tends toward 0 from (39) and (41). And

$$
\mathbf{A}\left(u_{n}, \mathscr{B}\left(u_{n}\right), \nabla w\right) \rightarrow \mathbf{A}(u, \mathscr{B}(u), \nabla w) \quad \text { strongly in } L^{q}(Q) .
$$

Thus

$$
\begin{aligned}
\liminf _{n \rightarrow \infty}\left(\mathbf{A}\left(u_{n}, \mathscr{B}\left(u_{n}\right), \nabla u_{n}\right), \nabla\left(u_{n}-v\right)\right) & \geqslant \frac{1}{\theta}(\mathbf{A}(u, \mathscr{B}(u), \nabla w), \nabla(u-w)) \\
& \geqslant(\mathbf{A}(u, \mathscr{B}(u), \nabla w), \nabla(u-v)) .
\end{aligned}
$$

Let $\theta$ go to 0 , we have owing to (A1),

$$
\liminf _{n \rightarrow \infty}\left(\mathbf{A}\left(u_{n}, \mathscr{B}\left(u_{n}\right), \nabla u_{n}\right), \nabla\left(u_{n}-v\right)\right) \geqslant(\mathbf{A}(u, \mathscr{B}(u), \nabla u), \nabla(u-v)) .
$$

Now we write

$$
\begin{aligned}
\left(\mathscr{A}\left(u_{n}\right), u_{n}-v\right)= & \left(\mathbf{A}\left(u_{n}, \mathscr{B}\left(u_{n}\right), \nabla u_{n}\right), \nabla\left(u_{n}-v\right)\right) \\
& +\left(A_{0}\left(u_{n}, \mathscr{B}\left(u_{n}\right), \nabla u_{n}\right), u_{n}-v\right) .
\end{aligned}
$$

Since

$$
\begin{aligned}
& \left(A_{0}\left(u_{n}, \mathscr{B}\left(u_{n}\right), \nabla u_{n}\right), u_{n}-v\right) \\
& \quad=\left(A_{0}\left(u_{n}, \mathscr{B}\left(u_{n}\right), \nabla u_{n}\right), u_{n}-u\right)+\left(A_{0}\left(u_{n}, \mathscr{B}\left(u_{n}\right), \nabla u_{n}\right), u-v\right)
\end{aligned}
$$

we have from (42) and (44)

$$
\left(A_{0}\left(u_{n}, \mathscr{B}\left(u_{n}\right), \nabla u_{n}\right), u_{n}-v\right) \rightarrow\left(A_{0}(u, \mathscr{B}(u), \nabla u), u-v\right),
$$

so that using (47) in (48) we conclude

$$
\liminf _{n \rightarrow \infty}\left(\mathscr{A}\left(u_{n}\right), u_{n}-v\right) \geqslant(\mathscr{A}(u), u-v) .
$$

That ends our proof. 
Remark 5. The crucial point is to derive (37) from (ii). To get a larger class of operators, one could study under which conditions the convergence

$$
\mathscr{B}\left(u_{n}\right) \rightarrow \mathscr{B}(u) \quad \text { strongly in } L^{q}(Q)
$$

implies

$$
u_{n} \rightarrow u \quad \text { strongly in } L^{p}(Q) .
$$

In this direction, Kačur uses a lemma ([14, Proposition 3.35]) which does not seem to generalize to our case. The strict convexity conditions of Visintin could also be used $[24,25]$.

For $f \in L^{q}(Q), g \in L^{q}\left(\Sigma_{2}\right)$, let $F \in L^{q}\left(0, T ; V^{\prime}\right)$ be defined by

$$
(F, v)=\int_{Q} f(x, t) v(x, t) \mathrm{d} x \mathrm{~d} t+\int_{\Sigma_{2}} g(\sigma) v(\sigma) \mathrm{d} \sigma, \quad \forall v \in L^{p}(0, T ; V) .
$$

For $u_{0} \in V$, we obtain existence of a solution $u$ verifying

$$
\frac{\mathrm{d}}{\mathrm{d} t} \mathscr{B}(u)+\mathscr{A}(u)=F \quad \text { and } \quad \mathscr{B}(u)(0)=\mathscr{B}\left(u^{0}\right),
$$

which is formally interpreted as

$$
\begin{aligned}
& \frac{\partial \beta(x, u)}{\partial t}-\operatorname{div} \mathbf{A}(x, t, u, \beta(u), \nabla u)+A_{0}(x, t, u, \beta(u), \nabla u)=f, \\
& u=0 \quad \text { on } \Sigma_{1}, \\
& \mathbf{A}(x, t, u, \beta(u), \nabla u) \cdot \mathbf{n}=g \quad \text { on } \Sigma_{2}, \\
& \beta(x, u(x, 0))=\beta\left(x, u_{0}(x)\right) \quad \text { on } \Omega .
\end{aligned}
$$

Let us mention that this result is in some sense better then those of [1] since our elliptic operator depends explicitly on $u$, and is not strongly monotone.

\section{Appendix A}

The following proof is influenced by those of [1].

Proof of Lemma 5. We write

$$
\begin{aligned}
& \int_{0}^{T-h}\left\|v_{\varepsilon}(t+h)-v_{\varepsilon}(t)\right\|_{W^{\prime}}^{q} \mathrm{~d} t \\
& \quad=\int_{E_{\varepsilon}^{M}}\left\|v_{\varepsilon}(t+h)-v_{\varepsilon}(t)\right\|_{W^{\prime}}^{q} \mathrm{~d} t+\int_{\left(E_{\varepsilon}^{M}\right)^{c}}\left\|v_{\varepsilon}(t+h)-v_{\varepsilon}(t)\right\|_{W^{\prime}}^{q} \mathrm{~d} t,
\end{aligned}
$$

where

$$
\begin{aligned}
E_{\varepsilon}^{M}= & \{t \in] 0, T-h\left[:\left\|u_{\varepsilon}(t)\right\|_{V}+\left\|u_{\varepsilon}(t+h)\right\|_{V}\right. \\
& \left.+\frac{1}{h^{1 / p}}\left\langle v_{\varepsilon}(t+h)-v_{\varepsilon}(t), u_{\varepsilon}(t+h)-u_{\varepsilon}(t)\right\rangle>M\right\} .
\end{aligned}
$$


First we bound the integral on $E_{\varepsilon}^{M}$.

We have

$$
\exists C>0, \forall \varepsilon>0, \quad \operatorname{meas}\left(E_{\varepsilon}^{M}\right) \leqslant \frac{C}{M} .
$$

Indeed,

$$
\begin{aligned}
\operatorname{meas}\left(E_{\varepsilon}^{M}\right)=\int_{E_{\varepsilon}^{M}} 1 \mathrm{~d} t \leqslant & \int_{E_{\varepsilon}^{M}} \frac{\left\|u_{\varepsilon}(t)\right\|_{V}}{M}+\frac{\left\|u_{\varepsilon}(t+h)\right\|_{V}}{M} \\
& +\frac{1}{M h^{1 / p}}\left\langle v_{\varepsilon}(t+h)-v_{\varepsilon}(t), u_{\varepsilon}(t+h)-u_{\varepsilon}(t)\right\rangle \mathrm{d} t .
\end{aligned}
$$

We have to bound $\left\|v_{\varepsilon}(t+h)-v_{\varepsilon}(t)\right\|_{W^{\prime}}^{q}$, which comes from estimation (iii).

Let $\eta>0$. We can find $M_{0}>0$ such that:

$$
\left.\forall M>M_{0}, \forall h \in\right] 0, T\left[, \quad \int_{E_{\varepsilon}^{M}}\left\|v_{\varepsilon}(t+h)-v_{\varepsilon}(t)\right\|_{W^{\prime}}^{q} \mathrm{~d} t \leqslant \eta .\right.
$$

In order to obtain our estimate to bound the integral on $\left(E_{\varepsilon}^{M}\right)^{c}$, we use the following adapted version of a lemma of [1]:

Lemma 6. Assume that $B$ is continuous from $W$ to $W^{\prime}$. Let $M>0$, and $\eta>0$. There exists $\delta>0$ such that for all $\left(u_{1}, u_{2}\right) \in V \times V$,

(i) $\left\|u_{i}\right\|_{V} \leqslant M, i=1,2$,

(ii) $\left\langle B\left(u_{1}\right)-B\left(u_{2}\right), u_{1}-u_{2}\right\rangle \leqslant \delta$

imply

$$
\left\|B\left(u_{1}\right)-B\left(u_{2}\right)\right\|_{W^{\prime}} \leqslant \eta .
$$

We use this lemma whose proof is given below.

We have $\forall t \in\left(E_{\varepsilon}^{M}\right)^{c}$,

$$
\begin{aligned}
& \left\|u_{\varepsilon}(t)\right\|_{V} \leqslant M, \quad\left\|u_{\varepsilon}(t+h)\right\|_{V} \leqslant M, \quad \text { and } \\
& \quad\left\langle v_{\varepsilon}(t+h)-v_{\varepsilon}(t), u_{\varepsilon}(t+h)-u_{\varepsilon}(t)\right\rangle \leqslant M h^{1 / p} .
\end{aligned}
$$

Thus there exists $h_{0}^{M}>0$, such that

$$
\forall h<h_{0}^{M}, \forall \varepsilon>0, \forall t \in\left(E_{\varepsilon}^{M}\right)^{c}, \quad\left\|v_{\varepsilon}(t+h)-v_{\varepsilon}(t)\right\|_{W^{\prime}}^{q} \leqslant \frac{\eta}{T} .
$$

Integrating on $\left(E_{\varepsilon}^{M}\right)^{c}$ we have

$$
\forall h\left\langle h_{0}^{M}, \forall \varepsilon\right\rangle 0, \quad \int_{\left(E_{\varepsilon}^{M}\right)^{c}}\left\|v_{\varepsilon}(t+h)-v_{\varepsilon}(t)\right\|_{W^{\prime}}^{q} \leqslant \eta .
$$

We deduce that there exists $M_{0}>0$ such that for all $M>M_{0}$ and for all $h<h_{0}^{M}$,

$$
\int_{0}^{T-h}\left\|v_{\varepsilon}(t+h)-v_{\varepsilon}(t)\right\|_{W^{\prime}}^{q} \mathrm{~d} t \leqslant 2 \eta
$$

This is exactly (29).

Proof of Lemma 6. If the conclusion of this lemma was false, there would exist $M>0$ and a sequence $\left\{u_{1, n}, u_{2, n}\right\}$ such that 
(i) $\left\|u_{i, n}\right\|_{V} \leqslant M$,

(ii) $\left\langle B\left(u_{1, n}\right)-B\left(u_{2, n}\right), u_{1, n}-u_{2, n}\right\rangle \leqslant \frac{1}{n}$

and

$$
\left\|B\left(u_{1, n}\right)-B\left(u_{2, n}\right)\right\|_{W^{\prime}} \geqslant \kappa>0 .
$$

As $\left(u_{i, n}\right)$ is bounded in $V$, and $B$ continuous on $W$, we can assume that up to a subsequence $B\left(u_{i, n}\right) \rightarrow v_{i}$ strongly in $W^{\prime}$.

Passing to the limit we would have

$$
\left|v_{2}-v_{1}\right|_{W^{\prime}} \geqslant \kappa>0 \text {. }
$$

But passing to the limit in (ii) gives

$$
\left\langle v_{1}-v_{2}, u_{1}-u_{2}\right\rangle=0,
$$

and the convexity of $\Phi$,

$$
\Phi \circ i\left(u_{2}\right)-\Phi \circ i\left(u_{1}\right) \leqslant\left\langle v_{2}, u_{2}-u_{1}\right\rangle \quad \text { et } \Phi \circ i\left(u_{2}\right)-\Phi \circ i\left(u_{1}\right) \geqslant\left\langle v_{1}, u_{2}-u_{1}\right\rangle .
$$

Thus

$$
\Phi \circ i\left(u_{2}\right)-\Phi \circ i\left(u_{1}\right)=\left\langle v_{1}, u_{2}-u_{1}\right\rangle=\left\langle v_{2}, u_{2}-u_{1}\right\rangle .
$$

Picking an arbitrary $z \in V$, we would have

$$
\begin{aligned}
\Phi \circ i\left(u_{2}+z\right)-\Phi \circ i\left(u_{2}\right) & =\Phi \circ i\left(u_{2}+z\right)-\Phi \circ i\left(u_{1}\right)-\left\langle v_{1}, u_{2}-u_{1}\right\rangle \\
& \geqslant\left\langle v_{1}, u_{2}+z-u_{1}\right\rangle-\left\langle v_{1}, u_{2}-u_{1}\right\rangle \\
& =\left\langle v_{1}, z\right\rangle .
\end{aligned}
$$

That would mean that $v_{1}=B\left(u_{2}\right)=v_{2}$, which contradicts $\left|v_{2}-v_{1}\right|_{W^{\prime}} \geqslant \kappa>0$.

\section{References}

[1] H.W. Alt, S. Luckhaus, Quasilinear elliptic-parabolic differential equations, Math. Z 183 (1983) 311-341.

[2] E.J. Balder, On weak convergence implying strong convergence under extremal conditions, J. Math. Anal. Appl. 163 (1992) 147-156.

[3] E.J. Balder, From weak to strong convergence in $L_{1}$ spaces via $K$-convergence, Ann. Mat. Pura Appl. CLXV(IV) (1993) 337-349.

[4] C. Bardos, H. Brézis, Sur une classe de problèmes d'évolution non linéaires, C. R. Acad. Sci. Paris. Sér. I Math. 266 (1968) 56-59.

[5] Ph. Bénilan, P. Wittbold, On mild and weak solutions of elliptic-parabolic problems, Adv. Differential Equations 1 (1996) 1053-1073.

[6] Ph. Bénilan, P. Wittbold, Sur un problème parabolique-elliptique, Math. Modelling Numer. Anal. (M2AN) 33 (1) (1999) 121-127.

[7] A. Bermúdez, J. Durany, C. Saguez, An existence theorem for an implicit nonlinear evolution equation, Collect. Math. (Barcelona) 35 (1) (1984) 19-34.

[8] H. Brézis, Equations et Inéquations non Linéaires dans les Espaces Vectoriels en Dualité, Ann. Inst. Fourier (Grenoble) 18 (1) (1968) 115-175.

[9] H. Brézis, Opérateurs Maximaux Monotones et Semi-Groupes de Contractions dans les Espaces de Hilbert, Number 5 in North-Holland Mathematics Studies, North-Holland Publishing Company, Amsterdam, 1973. 
[10] P. Colli, On Some Doubly Nonlinear Evolution Equations in Banach Spaces, Technical Report 775, Università di Pavia, Istituto di Analisi Numerica, 1991.

[11] E. DiBenedetto, R.E. Showalter, Implicit Degenerate Evolution Equations and Applications, SIAM J. Math. Anal. 12 (5) (1981) 731-751.

[12] J. Filo, J. Kačur, Local existence of General Nonlinear Parabolic Systems, Nonlinear Anal. 24 (11) (1995) 1597-1618.

[13] O. Grange, F. Mignot, Sur la Résolution d'une Équation et d'une Inéquation Paraboliques non Linéaires, J. Funct. Anal. 11 (1972) 77-92.

[14] J. Kačur, On a Solution of Degenerate Elliptic-Parabolic Systems in Orlicz-Sobolev Spaces, Math. Z 203 (1990) 153-171.

[15] M.A. Krasnoselskii et al., Integral Operators in Spaces of Summable Functions, Monographs and Textbooks on Mechanics of Solids and Fluids, Noordhoff International Publishing, Leiden, 1976.

[16] J.L. Lions, Quelques Méthodes de Résolution des Problèmes aux Limites Non Linéaires, Dunod, Paris, 1969.

[17] I. Maillot, Simulation Numérique du Remplissage-Compactage pour l'Injection des Thermo-plastiques, Thèse, Université Joseph Fourier, 1993.

[18] E. Maitre, Sur une classe d'équations à double non linéarité: application à la simulation numérique d'un écoulement visqueux compressible, Thèse, Université Grenoble I, 1997.

[19] E. Maitre, P. Witomski, Equation à double non-linéarité par régularisation parabolique. Preprint RT140, Laboratoire de Modélisation et Calcul LMC-IMAG, Université de Grenoble I, 1995.

[20] P.A. Raviart, Sur la Résolution de Certaines Équations Paraboliques non Linéaires, J. Funct. Anal. 5 (1970) 299-328.

[21] R.T. Rockafellar, Convex integral functionals and duality, in: E. Zaratonello (Ed.), Contributions to Nonlinear Functional Analysis, Academic Press, 1971, pp 215-236.

[22] J. Simon, Compact Sets in the Space $L^{p}(0, T ; B)$, Ann. Mat. Pura Appl. 146 (1987) 65-96.

[23] M.M. Vainberg, Variational Methods for the Study of Nonlinear Operators, Mathematical Physics, Holden-Day, San Fransisco, CA, 1964.

[24] A. Visintin, Strong Convergence results related to strict convexity, Commun. Partial Differential Equations 9 (5) (1984) 439-466.

[25] A. Visintin, Models of phase transitions, Birkhäuser, Boston, 1996.

[26] E. Zadrzyńska, W.M. Zajączkowski, On existence of solutions of mixed problems for parabolic systems, Topol. Method Nonlinear Anal. 2 (1993) 125-145.

[27] E. Zeidler, Nonlinear functional analysis and its application, Vol. I-IV, Springer, Berlin, 1988. 